\title{
Analysis of the $(s, S)$ policy for perishables with a random shelf life
}

\author{
ÜLKÜ GÜRLER ${ }^{1, *}$ and BANU YÜKSEL ÖZKAYA ${ }^{2}$ \\ ${ }^{1}$ Department of Industrial Engineering, Bilkent University, Ankara, Turkey \\ E-mail:ulku@bilkent.edu.tr \\ ${ }^{2}$ Department of Industrial Engineering, Hacettepe University, Ankara, Turkey
}

Received June 2006 and accepted July 2007

\begin{abstract}
A continuous review perishable inventory system operating under the $(s, S)$ policy is considered. Assuming a random shelf life with a general distribution, renewal arrivals and a negligible replenishment lead time, exact expressions for the expected cost rate function for unit and batch demands are derived. For unit demands, it is shown that the average cost rate function is quasi-convex in ( $s, S$ ). Numerical findings indicate that the loss incurred by ignoring the randomness of the shelf life can be drastic. It is observed that the shape of the shelf life distribution has a significant impact on the costs and a precise estimation of shelf life distribution may result in substantial savings. Based on the presented analytical results, a new heuristic for positive lead times is proposed. Extensive numerical studies show that the proposed heuristic performs better than an existing one suggested for fixed shelf lives in most of the cases studied.
\end{abstract}

Keywords: Perishable, random shelf life, batch demand, renewal arrival.

\section{Introduction and literature review}

Most of the existing models in the inventory literature assume that items have an infinite shelf life and thus can be stored indefinitely. This assumption is not realistic for perishable goods that can deteriorate and become unusable after some finite time. Fresh food stuff, blood products, meat, chemicals, composite materials and pharmaceuticals are all examples of perishable products.

Perishability is generally modeled in one of three ways.

1. Continuous deterioration, where the items in stock decay with a rate proportional to the amount and/or age of the items. Volatile chemicals and radioactive materials are examples of this type of decay.

2. Independent random shelf lives of individual items in stock. In this setting, all of the items have identical shelf life distributions but they perish (i.e. become unusable) individually. Only the exponential shelf life is considered in the literature for this case. Although the memoryless property of the exponential distribution and independent degradation of individual items are convenient for the analysis, this model may not be appropriate for products whose lifetimes are correlated due to external factors, such as storage conditions or the internal dynamics of the supply chain.

${ }^{*}$ Corresponding author
3. Batches of items having the same shelf lives, where the items in a single batch perish at the same time. The shelf life itself may be constant or random.

In this study, we consider the last degradation type with items in the same batch having the same random lifetime with a general distribution.

Consider the following example, communicated by a colleague with experience in the distribution of apples to grocery stores. Each year's harvest is stored in very large batches in sealed, low-oxygen and low-temperature cells. Apples can be stored in such sealed cells for very long periods of time; however, once a cell is unsealed, the apples have a remaining shelf life of about 6 weeks. Over time, the sealed cells are opened and their contents are used to satisfy orders placed by individual grocery stores. Practically, each individual order is satisfied by the material stored in the most recently opened cell; hence, all of the produce within the replenishment order come from the same vintage and have the same shelf life at the store level. As orders arrive according to different consumption patterns at individual stores, the remaining shelf lives of the batches as experienced by an individual store are random, although each sealed cell has a fixed shelf life itself. (See also Johnston et al. (2002) for issues regarding the post-harvest storage factors that influence the perishability of the apples.)

This example can be formalized and generalized to all perishable items with a fixed shelf life at the upper 

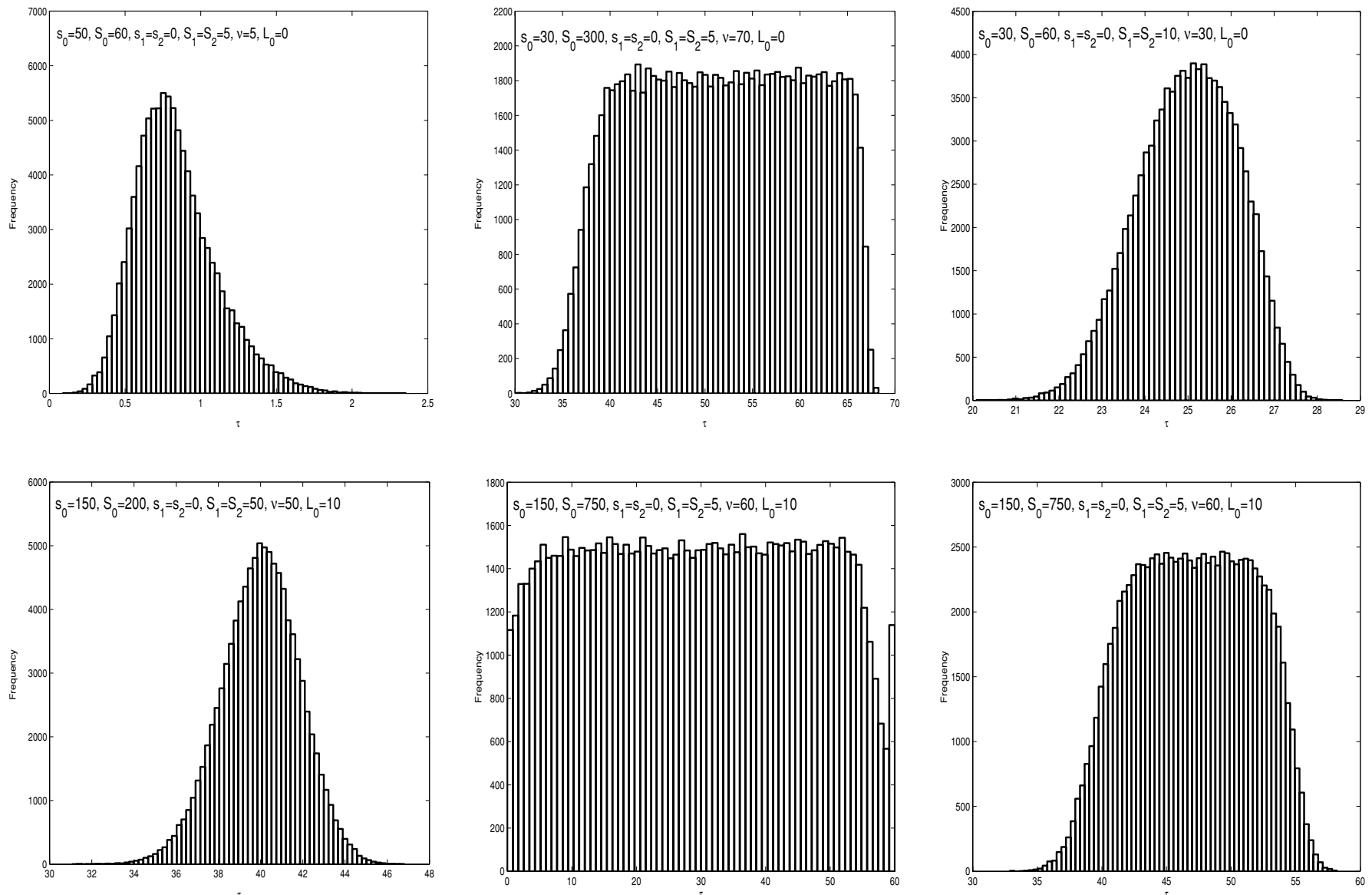

Fig. 1. Simulated frequency distribution of the shelf life faced by the retailer in a single-warehouse two-retailer system.

echelon in a supply chain. We provide a simulated example below. Consider a continuous review two-echelon inventory system for a perishable item, with a single warehouse and a number of identical retailers. Suppose that the retailers face independent unit renewal demands and the warehouse orders from an outside supplier with a constant lead time. Assume that the items arrive at the warehouse fresh, with a fixed shelf life $v$. Suppose also that the shipment time from the warehouse to the retailers is negligible and all retailers employ an $(s, S)$-type control policy. For convenience, assume that the warehouse also employs an $(s, S)$-type policy. Then, the items in a batch shipped from the warehouse to the retailer will have the same random shelf life which is characterized by the steady-state distribution of the remaining shelf life of the items in stock at the warehouse level. A set of examples for the simulated steady-state distributions of the shelf life faced by the retailer is illustrated in Fig. 1 for a single-warehouse two retailer-system, under different system parameters. (In the graphs, the subscripts $0,1,2$ refer to the warehouse, the first and the second retailers respectively and $L_{0}$ stands for the warehouse replenishment lead time.) We observe that, depending on the particulars of the system parameters, batches that arrive at a retailer may assume different shelf life distributions. Hence, we find it interesting and important to study the impact of shelf life distributions on replenishment decisions and inventory system performance for perishable goods.

In this study, we consider a model for perishable goods at the retailer level, similar to the one discussed in the above setting. More specifically, we consider the replenishment of a perishable product under a continuous review $(s, S)$ policy with renewal batch demands (both discrete and continuous demand sizes) and negligible replenishment lead times. We assume that the shelf life of the items is a random variable and that items in the same batch perish at the same time. On the other hand, the shelf life of items in different batches are independent and identically distributed. Before we present the details of our model, we briefly review the related literature.

Continuously deteriorating inventory systems are extensively investigated in the literature, and interested readers are referred to the review papers by Raafat (1991) and Goyal and Giri (2001). The literature on the case in which each item in a batch independently perishes is limited to exponential lifetimes. Kalpakam and Arivarignan (1988) study a continuous review $(s, S)$ model with Poisson demand and zero lead time. Liu (1990) allows backorders for the same model. Moorthy et al. (1992) propose another 
aging structure in which the shelf life of an item starts when it is put on display after the previous item is sold or expires. Kalpakam and Sapna (1994) and Liu and Yang (1999) consider extensions of the Kalpakam-Arivarignan model, whereas Kalpakam and Sapna (1996) discuss a lost-sales $(S-1, S)$ policy with exponential lead times and renewal demands. Liu and Shi (1999) study an $(s, S)$ model with an exponential shelf life, where degradation is only detected at demand arrivals. Recently, Kalpakam and Shanthi (2001) analyze a lost sales, Poisson demand model with an exponential shelf life and a random lead time operating under an $(S-1, S)$ policy.

Early studies on perishable goods assume a periodic review approach with a fixed shelf life. Van Zyl (1964) provides the first finite-horizon and infinite-horizon dynamic programming formulations with a shelf life of exactly two periods. Nahmias and Pierskalla (1975) investigate the properties of the optimal policy for two-period shelf lives and Fries (1975) and Nahmias (1975) extend the results to $m$ periods. Because the optimal policy structure is very complex, for longer shelf lives, approximate policies have been developed by Cohen (1976) and Nahmias (1976, 1977a). Nahmias (1977b) considers extensions to the random shelf life approach and establishes the similarity of the optimal policy structure to that for a fixed shelf life. A comprehensive survey of the studies with periodic review can be found in Nahmias (1982).

The current literature on continuous review perishable problems considers various assumptions on lead times. With a zero lead time, Weiss (1980) shows that an $(s, S)$ type policy is optimal for unit Poisson demand and constant shelf life. Liu and Lian (1999a) consider a continuous review $(s, S)$ model with a fixed shelf life and renewal arrivals. Liu and Lian (1999b) assume a zero lead time and allowing for backorders, they study an $(s, S)$ model with geometric inter-demand times. Lian and Liu (2001) study an $(s, S)$ policy with a fixed shelf life and renewal batch demands. Using Markov chain methods and Laplace-Steltjes transforms, they provide an iterative approach for finding the expected length of a regenerative cycle and present expected sojourn times through which the expected holding and backorder cost of a cycle is obtained (see also Gürler and Özkaya (2003) for a correction of Lian and Liu (2001). With zero or exponential lead times, it is usually possible to analyze a perishable inventory system using Markov renewal techniques. A constant positive lead time however, does not allow a Markovian structure and the analysis becomes very complicated. Nahmias and Wang (1979) provide the first approximate analysis of a perishable inventory system with a constant lead time and the first exact study of an $(S-1, S)$ policy with a fixed shelf life, constant lead time and lost sales assumption is given by Schmidt and Nahmias (1985). Later Chiu (1995) proposed an approximation for the continuous review $(Q, r)$ model with a fixed shelf life. Perry and Posner (1998) study an $(S-1, S)$ policy with a fixed shelf life, Poisson demand and lead-time- dependent backordering. Ravichandran (1995) considers an $(s, S)$ inventory model with a random lead time and Poisson demand with a specific aging pattern. Tekin et al. (2001) extend the work of Ravichandran (1995) by considering a time based policy when the lead time is a positive constant.

Our study generalizes and improves on the previous work in several aspects. First, the proposed model allows the shelf life to be a random variable, which, apart from being applicable to items with inherently random shelf lives, also allows the treatment of fixed shelf life products that may perish randomly due to imperfect storage conditions. In this respect, we also extensively investigate the impact of the shelf life distribution and obtain practically important findings. To the best of our knowledge only the exponential distribution has been considered in the literature. Second, we present explicit expressions for the expected cost rate and the operating characteristics for the model studied, from which the explicit cost expressions of the models by Liu and Lian (1999) and Lian and Liu (2001) can be obtained. We also show that the expected cost rate function is quasi-convex in $(s, S)$ for unit demand, which guarantees unimodality and facilitates finding the global optimum in a numerical search. Finally, based on the analytical findings for the zero lead time case, we provide a heuristic for positive lead times.

The results of our extensive numerical study indicate that the loss incurred by ignoring the randomness of the shelf life can be substantial. We observe that treating the shelf life as being constant when it is random results in 21.42 and $5.90 \%$ average losses for unit and gamma demands respectively. Including the unreported cases with other demand rates and coefficient of variances for the shelf life, the average losses become as high as $23.10,9.26$, and $9.45 \%$ for unit, geometric and gamma demands respectively, over the 104 cases studied. We also observe that the distribution of the shelf life has a significant impact on the system costs, and the average cost differences can get as large as 20,13 and 13\% for unit, geometric and gamma demands. In unreported results, we observed that the highest costs are observed for the exponential shelf life case. We note that the proposed heuristic performs as good as or better than the existing one by Lian and Liu (2001) suggested for fixed shelf life studies in most of the cases, although on the average the existing heuristic performs slightly better for the unit demand. In particular, the deviation of the proposed heuristic from the simulated exact model is 0.34 and $1.60 \%$ for the unit and the batch demands over the 48 cases, whereas the corresponding figures are 0.29 and $3.62 \%$ for the existing heuristic of Lian and Liu (2001).

The rest of the paper is organized as follows. In Section 2 , we introduce the model and derive the operating characteristics for both discrete and continuous batch demands. In Section 3, analytical results for the unit demand case are presented. In Section 4, a heuristic is developed for positive lead times and in Section 5, results of the extensive 
numerical study are presented. The paper ends with concluding remarks in Section 6.

\section{Model and analysis}

We consider a single-item single-location inventory system in which the items face batch demands that arrive according to a renewal process with random batch sizes that are independent of the arrival process. Unmet demands are backordered and if the entire demand can not be satisfied by the on-hand stock, it is partially filled immediately with the available stock and the rest is backordered. The shelf life of the products, $\tau$, is a random variable with a general distribution. We assume that products in an arriving order have the same shelf life, where the shelf lives of items in different orders are independent and identically distributed random variables. The costs associated with the inventory system are the fixed ordering cost $K$ per order; the holding cost $h$ per unit per time; the degradation cost $\pi$ per unit; the backorder cost $b$ per unit and $\rho$ per unit per time. The replenishment lead time, $L$, is assumed to be zero and the following continuous review $(s, S)$ ordering policy is used:

Policy: When the inventory level drops to s or below, a replenishment order is placed to raise it up to $S$.

For unit demands, Weiss (1980) shows that the optimal replenishment policy for this model is of $(s, S)$ type when backorder costs are increasing convex in the time until satisfaction and the replenishment lead time is zero. The zero replenishment lead time assumption induces an upper bound on $s$ as $s<0$, since, otherwise the instantaneously arriving fresh items wait for the next demand, during which they incur a holding cost and are at risk of degradation while they are held in stock. Also, if $S<0$, the inventory level is always negative, items never perish and the model becomes mathematically trivial and practically uninteresting. We therefore assume that $S \geq 0$.

Under the above policy, the instances at which the inventory level is raised to $S$ constitute regeneration points and a regenerative cycle is defined as the time between two such consecutive instances. For the derivation of the operating characteristics, we partition a regenerative cycle into two segments. The first one, subcycle 1 is the time from the beginning of a cycle until the inventory level becomes negative for the first time and the second one, subcycle 2 is the remaining time to complete the cycle. If at the end of subcycle 1 , the inventory level is below $s$, then an order is placed immediately which completes the cycle and subcycle 2 is not realized.

We use the following notation in our analysis: $N(t)$ is the counting process of the demand arrivals up to time $t$, where $t=0$ is taken as the beginning of a regenerative cycle. The inter-arrival times are independent and identically distributed random variables denoted by $X$, with mean $\mu$. $\left\{X_{n}, n=1,2, \ldots\right\}$ is the sequence of arrival times since the beginning of a cycle, with distribution function (d.f.) $F_{n}$ and $\left\{d_{i}, i \geq 1\right\}$ is the sequence of batch sizes with d.f. $V$. Also let $D_{k}=\sum_{i=1}^{k} d_{i}$ denote the cumulative demand at the $k$ th arrival with d.f. $V_{k}$ and $G$ be the d.f. of the random shelf life $\tau$. We define $l_{0}$ as the number of demand arrivals by which the inventory level drops below zero for the first time and $r_{1}$ as the level of the inventory when it drops below zero for the first time. In what follows, for a d.f. $F, \bar{F}=1-F$, the function $I(\cdot)$ is the indicator of its argument and the conventions $D_{0}=X_{0}=0$ and $F_{0}(x)=V_{0}(x)=1$ for $x \geq 0$, zero otherwise are used.

Objective function: In view of the renewal reward theorem (Ross (1983)), the optimization problem is stated as the minimization of the ratio of the expected cycle cost to that of cycle length. That is

$$
\min _{s, S} A C(s, S)=\frac{E[C C(s, S)]}{E[C L(s, S)]},
$$

where $A C(s, S), C C(s, S)$ and $C L(s, S)$ are the average cost rate, cycle cost and cycle length, respectively.

We first discuss below the discrete demand case, and extend the results to continuous demand in Section 2.2.

\subsection{Discrete demand}

We now consider the case where the demand batch size is a discrete random variable with probability mass function (p.m.f.) $v$, and $v_{k}$ is the p.m.f. of the cumulative demand at the $k$ th arrival since the beginning of a cycle.

Figure 2 illustrates some realizations for a cycle when the demand batch size is a discrete random variable. Case 1 refers to a realization where the inventory level drops exactly to zero by demand and no degradation occurs. In case 2 , items do not perish, and the inventory level crosses zero without staying at level zero, whereas in case 3 , some items in the batch perish before they are depleted by demand. In all three cases, the cycles are completed immediately when the inventory level hits or crosses $s$. We assume that when demand is partially fulfilled, a backorder cost is incurred only for the unsatisfied portion of the batch. Also note that since $L=0$, the demand batch which triggers the replenishment order is satisfied immediately and does not incur a backorder cost.

We start by presenting a result which provides the p.m.f. of $r_{1}$, the level of the inventory in a cycle when it first drops below zero.

Lemma 1. The p.m.f. of $r_{1}$ is given as below for $x=$ $-1,-2, \ldots$ :

$$
\begin{aligned}
& P_{r_{1}}(x)= \\
& v(-x) \sum_{k=0}^{S}\left[\int_{u=0}^{\infty}\left[V_{k}(S) F_{k}(u)-V_{k}(S-1) F_{k+1}(u)\right] \mathrm{d} G(u)\right] \\
& +\sum_{k=1}^{S} \int_{u=0}^{\infty} F_{k}(u) \mathrm{d} G(u)\left[\sum_{i=k-1}^{S-1} v_{k-1}(i) v(S-i-x)\right]
\end{aligned}
$$

Proof. See the Appendix. 


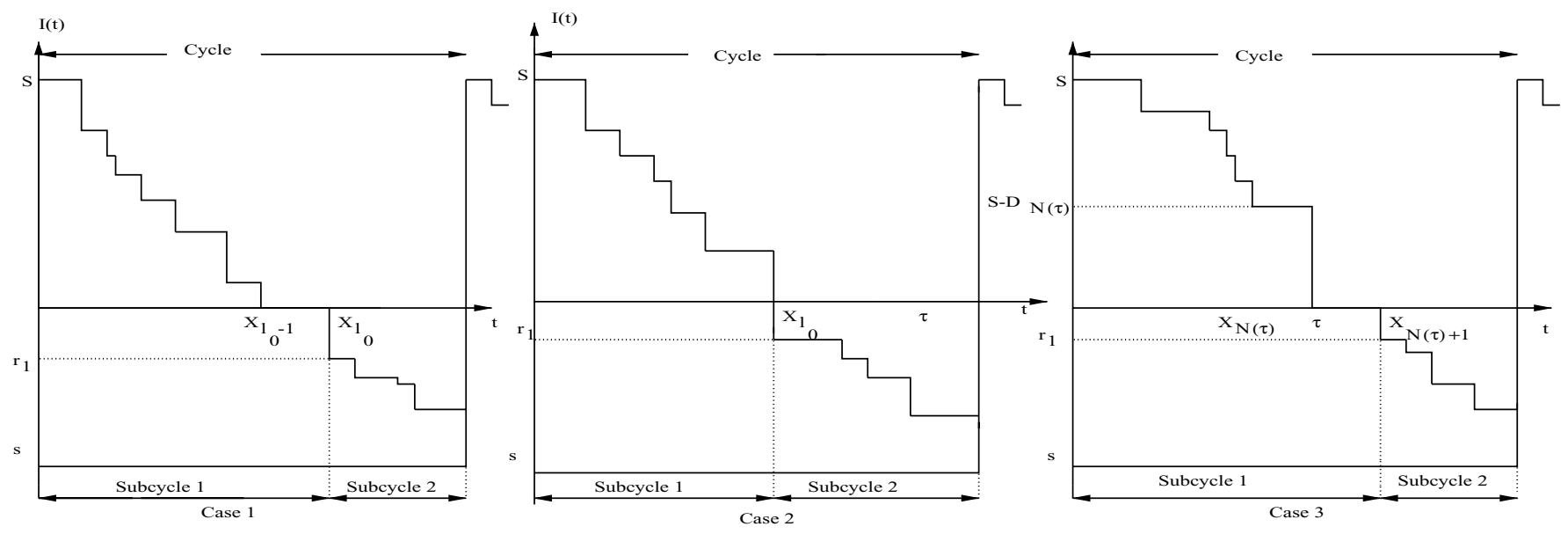

Fig. 2. Possible cycle realizations.

\subsubsection{Expected cycle length}

Recall that we have partitioned a regenerative cycle into two segments. Subcycle 1 is the time from the beginning of a cycle until the inventory becomes negative for the first time and subcycle 2 is the time from the end of subcycle 1 to the end of the regenerative cycle. Let $C L_{1}(s, S)$ and $C L_{2}(s, S)$ be the lengths of subcycles 1 and 2 respectively. Then,

$$
\begin{aligned}
& C L_{1}(s, S) \\
& = \begin{cases}X_{l_{0}} & \text { if } X_{l_{0}-1}<\tau, D_{l_{0}-1}=S \text { or } \\
& X_{l_{0}}<\tau, D_{l_{0}-1} \leq S-1, D_{l_{0}}>S \\
X_{N(\tau)+1} & \text { if } X_{N(\tau)}<\tau<X_{N(\tau)+1}, D_{N(\tau)} \leq S-1\end{cases}
\end{aligned}
$$

The first expression above corresponds to the cases where the inventory level drops exactly to zero by demands and stays there until the next demand arrives or becomes negative without staying at zero (cases 1 and 2 in Fig. 2) and the second one refers to degradation (case 3 in Fig. 2). Let $n_{2}$ denote the number of demand arrivals in subcycle 2 . To avoid confusion, we denote the arrival time of the $i$ th demand in subcycle 2 by $\tilde{X}_{i}$ and the cumulative demand at the $i$ th demand arrival by $\tilde{D}_{i}$, which are identically distributed with $X_{i}$ and $D_{i}$ of subcycle 1 , respectively. Then, $C L_{2}(s, S)$ is written as

$$
\begin{aligned}
& C L_{2}(s, S) \\
& = \begin{cases}\tilde{X}_{n_{2}} & \text { if } \tilde{D}_{n_{2}-1} \leq r_{1}-s-1, \tilde{D}_{n_{2}} \geq r_{1}-s, r_{1}>s . \\
0 & \text { if } r_{1} \leq s .\end{cases}
\end{aligned}
$$

Taking the expectations of Equations (2) and (3) and using Lemma A1., we obtain the following, proofs of which are given in the Appendix.

$$
\begin{aligned}
& E\left[C L_{1}(s, S)\right]=\mu \sum_{k=0}^{S} V_{k}(S) \int_{x=0}^{\infty} F_{k}(x) \mathrm{d} G(x), \\
& E\left[C L_{2}(s, S)\right]=\mu \sum_{x=s+1}^{-1} \sum_{k=0}^{x-s-1} V_{k}(x-s-1) P_{r_{1}}(x) .
\end{aligned}
$$

The expected cycle length $E[C L(s, S)]$ is then obtained as the sum of $E\left[C L_{1}(s, S)\right]$ and $E\left[C L_{2}(s, S)\right]$.

\subsubsection{Expected cycle cost}

The derivation of the costs for the two subcycles will be considered separately. In subcycle 1 , a backorder cost is triggered only by the last demand if $r_{1}>s$. If $r_{1} \leq s$, subcycle 1 and the cycle are completed simultaneously with order placement and no shortage cost is incurred. Therefore, the unit-dependent shortage cost, $U S C^{1}$, of subcycle 1 is $-b r_{1} I\left(r_{1}>s\right)$ and

$$
E\left[U S C^{1}\right]=-b \sum_{x=s+1}^{-1} x P_{r_{1}}(x) .
$$

For the holding and perishing costs, suppose the inventory level is $S-D_{i-1} \geq 0$ after the $(i-1)$ th demand. Then, the next event will either be the arrival of the $i$ th demand (Figs. 3(a), 3(d) and 3(e)), if $X_{i}<\tau$ or perishing if $X_{i} \geq \tau$ (Fig. 3(b) and 3(c)). Then, the holding cost, $H C_{i}$, and the perishing cost, $P C_{i}$, associated with the $i$ th demand are given as

$$
\begin{aligned}
& H C_{i}= \begin{cases}h\left(X_{i}-X_{i-1}\right)\left(S-D_{i-1}\right) & \text { if } \quad X_{i}<\tau, \\
& D_{i-1}<S . \\
h\left(\tau-X_{i-1}\right)\left(S-D_{i-1}\right) & \text { if } \quad \begin{array}{l}
X_{i-1}<\tau \leq X_{i}, \\
D_{i-1}<S .
\end{array}\end{cases} \\
& P C_{i}=\pi\left(S-D_{i-1}\right) I\left(X_{i-1}<\tau \leq X_{i}, D_{i-1}<S\right) .
\end{aligned}
$$

Taking expectations and summing over $i$, we find the expected holding cost, $E[H C(s, S)]$, and the expected perishing cost, $E[P C(s, S)]$, of the cycle as

$$
\begin{aligned}
E[H C(s, S)]= & h \sum_{i=1}^{S} \sum_{k=i-1}^{S-1} V_{i-1}(k) \\
& \times \int_{u=0}^{\infty}\left[F_{i-1}(u)-F_{i}(u)\right] \bar{G}(u) \mathrm{d} u,
\end{aligned}
$$




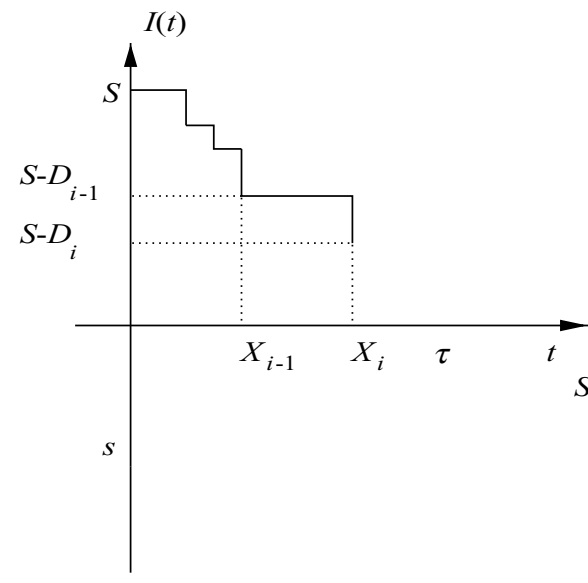

(a)

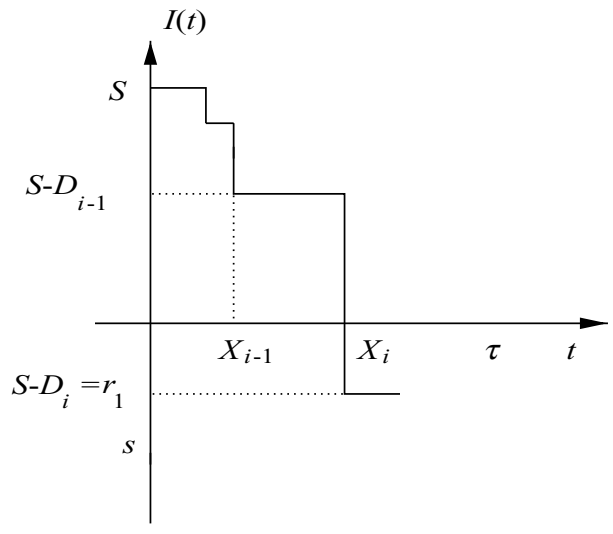

(d)

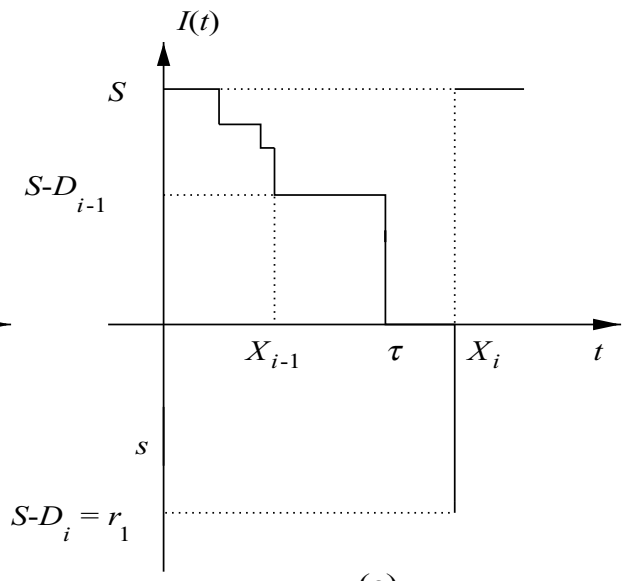

(c) (b)

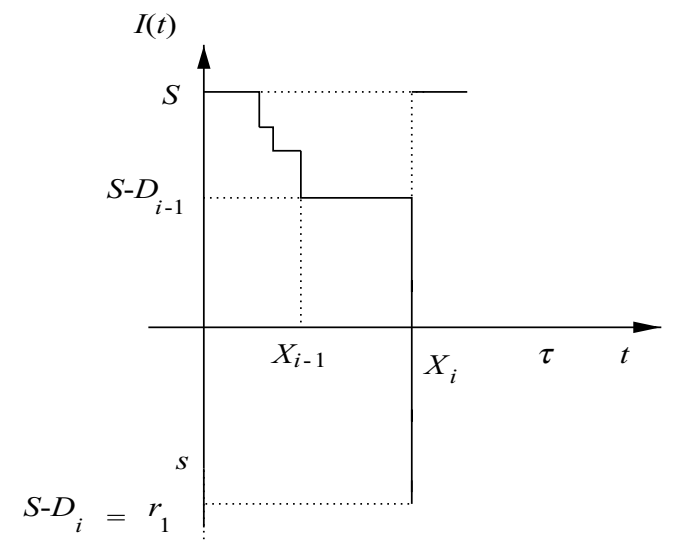

(e)

Fig. 3. Possible demand realizations. when $S-D_{i-1} \geq 0$.

$$
\begin{aligned}
E[P C(s, S)]= & \pi \sum_{i=1}^{S} \sum_{k=i-1}^{S-1} V_{i-1}(k) \\
& \times \int_{u=0}^{\infty}\left[F_{i-1}(u)-F_{i}(u)\right] \mathrm{d} G(u) .
\end{aligned}
$$

Now, we consider the costs incurred in subcycle 2.

Suppose the inventory level is $s<r_{1}-\tilde{D}_{i-1}<0$ after the $(i-1)$ th demand. Then, there may be two realizations: if the demand of the $i$ th arrival is $\tilde{d}_{i}$ and the inventory level drops to $r_{1}-\tilde{D}_{i-1}-\tilde{d}_{i}>s($ Fig. 4(a)), the cycle is not completed and a time-dependent shortage cost for $\tilde{D}_{i-1}-r_{1}$ backordered items is incurred as well as the unit-dependent shortage cost for the additional $\tilde{d}_{i}$ items backordered. Otherwise, $r_{1}-\tilde{D}_{i-1}-\tilde{d}_{i} \leq s$ and the cycle is completed (Fig. 4(b)), incurring only the time-dependent shortage cost for $\tilde{D}_{i-1}-r_{1}$ backordered items during $\left(\tilde{X}_{i-1}, \tilde{X}_{i}\right)$. In fact, the unit shortage costs are incurred for all the backordered units in subcycle 2, except for the last arrival which ends the cycle. Let $U S C_{i}^{2}$ be the unit shortage cost of subcycle 2 if it terminates with the $i$ th demand arrival and similarly, let $T D S C_{i}$ denote the time-dependent shortage cost of subcycle 2 that terminates with the $i$ th demand. Then,

$$
U S C_{i}^{2}=b \tilde{D}_{i-1} \quad \text { if } \tilde{D}_{i-1} \leq r_{1}-s-1, \quad \tilde{D}_{i} \geq r_{1}-s,
$$

$T D S C_{i}=\rho\left(\tilde{X}_{i}-\tilde{X}_{i-1}\right)\left(\tilde{D}_{i-1}-r_{1}\right) \quad$ if $\tilde{D}_{i-1} \leq r_{1}-s-1$.

The expected unit-dependent shortage cost in subcycle 2 is

$$
\begin{aligned}
E & {\left[U S C^{2}\right] } \\
& =\sum_{x=s+1}^{-1} \sum_{i=1}^{x-s} E\left[U S C_{i}^{2} \mid x\right] P_{r_{1}}(x) \\
& =b \sum_{x=s+1}^{-1}\left[\sum_{i=1}^{x-s} \sum_{k=i-1}^{x-s-1} k \bar{V}(x-s-k-1) v_{i-1}(k)\right] P_{r_{1}}(x),
\end{aligned}
$$

and the sum of Equations (6) and (13) gives the total expected unit shortage cost as

$$
\begin{aligned}
& E[U S C(s, S)] \\
& =b \sum_{\substack{x=s+1\\
}}^{-1}\left[-x+\sum_{i=1}^{x-s} \sum_{k=i-1}^{x-s-1} k \bar{V}(x-s-k-1) v_{i-1}(k)\right] \\
& \quad \times P_{r_{1}}(x) .
\end{aligned}
$$




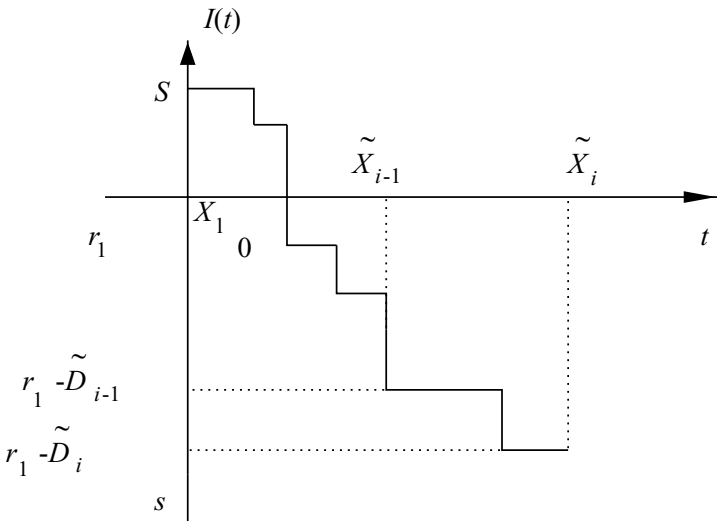

(a)

Fig. 4. Possible demand realizations when $S-D_{i-1}<0$.

The expected time-dependent shortage cost is written similarly as

$$
\begin{aligned}
E[T D S C(s, S)] & =\sum_{x=s+1}^{-1} \sum_{i=1}^{x-s} E\left[T D S C_{i} \mid x\right] P_{r_{1}}(x) \\
& =\rho \mu \sum_{x=s+1}^{-1} \sum_{i=1}^{x-s} \sum_{k=i-1}^{x-s-1}(k-x) v_{i-1}(k) P_{r_{1}}(x) .
\end{aligned}
$$

The expected cycle cost $E[C C(s, S)]$ is then obtained as the sum of the ordering cost $K$ and the expected holding, perishing, unit and time-dependent shortage costs given in Equations (9), (10), (14), and (15) respectively.

We next provide the operating characteristics of the special case where the shelf life is a constant.

2.1.2.1. Constant shelf life. If we let $G(t)=I(t \geq T)$, we obtain the model of Lian and Liu (2001), where the shelf life is a constant, $T$. In their results, the objective function is obtained by iteratively solving Laplace transforms. Below we present the explicit expressions for the operating characteristics of this special case:

$$
\begin{aligned}
P_{r_{1}}(x)= & v(-x) \sum_{k=0}^{S}\left[F_{k}(T) V_{k}(S)-F_{k+1}(T)\right. \\
& \left.\times V_{k}(S-1)\right]+\sum_{k=1}^{S} F_{k}(T) \\
& \times\left[\sum_{i=k-1}^{S-1} v_{k-1}(i) v(S-i-x)\right], \\
E[C L(s, S)]= & \mu\left[\sum_{k=0}^{S} V_{k}(S) F_{k}(T)+\sum_{x=s+1}^{-1} P_{r_{1}}(x)\right. \\
& \left.\times \sum_{k=0}^{x-s-1} V_{k}(x-s-1)\right],
\end{aligned}
$$

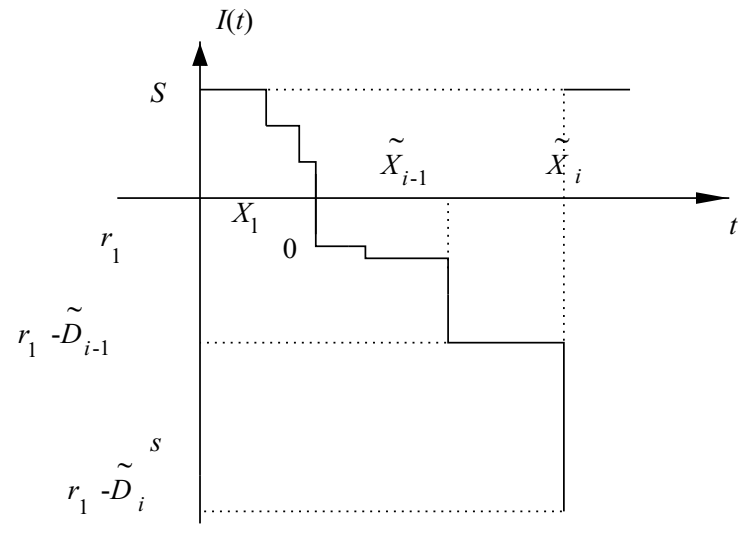

(b)

$$
\begin{aligned}
& E[H C(s, S)]=h \sum_{i=1}^{S} \sum_{k=i-1}^{S-1} V_{i-1}(k) \int_{u=0}^{T}\left[F_{i-1}(u)-F_{i}(u)\right] \mathrm{d} u, \\
& E[P C(s, S)]=\pi \sum_{i=1}^{S} \sum_{k=i-1}^{S-1} V_{i-1}(k)\left[F_{i-1}(T)-F_{i}(T)\right] .
\end{aligned}
$$

The expressions for the expected shortage costs of a cycle are as given in Equations (14) and (15).

\subsection{Continuous demand}

Next we extend our results to the continuous demand case. We retain the rest of the assumptions and the notation introduced before, except that $v($.$) now denotes the probability$ density function (p.d.f). of the continuous batch size of a demand. The level of the inventory when it first drops below zero, $r_{1}$, is now a continuous random variable with d.f. and p.d.f given by $F_{r_{1}}($.$) and f_{r_{1}}($.$) respectively. As to the$ differences between discrete and continuous demands, we note that due to the continuity of the batch size, the number of demand arrivals needed to deplete an inventory of say $S$ units is no longer bounded. Also, the probability that the inventory hits the zero level by demand arrivals and stays there a positive amount of time is zero, hence such events are no longer taken into account in the derivations. We present the key expressions below for the continuous demand case, the proofs of some of which are given in the Appendix. First, we present a result analogous to Lemma 1 for the p.d.f. of $r_{1}$.

Lemma 2. The p.d.f. of $r_{1}$ is given as follows for $x<0$ :

$$
\begin{aligned}
f_{r_{1}}(x)= & v(-x) \sum_{k=0}^{\infty} V_{k}(S) \int_{z=0}^{\infty}\left[F_{k}(z)-F_{k+1}(z)\right] \mathrm{d} G(z) \\
& +\sum_{k=1}^{\infty} \int_{z=0}^{\infty} F_{k}(z) \mathrm{d} G(z) \int_{u=0}^{S} v(S-x-u) \mathrm{d} V_{k-1}(u) .
\end{aligned}
$$


Proof. See the Appendix.

For the expected length of subcycle 1, consider Equation (2) and note that now the event that $\left\{D_{l_{0}-1}=S, X_{l_{0}-1}<\tau\right\}$ has a zero probability. For subcycle $2, C L_{2}(s, S)$ is written as given in Equation (3). Then,

$$
\begin{aligned}
& E\left[C L_{1}(s, S)\right]=\mu \sum_{k=0}^{\infty} V_{k}(S) \int_{x=0}^{\infty} F_{k}(x) \mathrm{d} G(x), \\
& E\left[C L_{2}(s, S)\right]=\mu \int_{x=s}^{0} \sum_{k=0}^{\infty} V_{k}(x-s) \mathrm{d} F_{r_{1}}(x) .
\end{aligned}
$$

Regarding the expected cycle cost, the modified expressions for continuous demand are given as below, the details of which are skipped since they are obtained similar to the discrete case.

$$
\begin{aligned}
E\left[H C_{i}\right] & =h \int_{x=0}^{S} V_{i-1}(x) \mathrm{d} x \int_{u=0}^{\infty}\left[F_{i-1}(u)-F_{i}(u)\right] \bar{G}(u) \mathrm{d} u, \\
E\left[P C_{i}\right] & =\pi \int_{x=0}^{S} V_{i-1}(x) \mathrm{d} x \int_{u=0}^{\infty}\left[F_{i-1}(u)-F_{i}(u)\right] \mathrm{d} G(u), \\
E\left[U S C^{1}\right] & =-b \int_{x=s}^{0} x \mathrm{~d} F_{r_{1}}(x), \\
E\left[U S C_{i}^{2}\right] & =b \int_{x=s}^{0} \int_{t=0}^{x-s} t \bar{V}(x-s-t) \mathrm{d} V_{i-1}(t) \mathrm{d} F_{r_{1}}(x), \\
E\left[T D S C_{i}\right] & =\rho \mu \int_{x=s}^{0} \int_{t=0}^{x-s}(t-x) \mathrm{d} V_{i-1}(t) \mathrm{d} F_{r_{1}}(x) .
\end{aligned}
$$

The final expressions are then obtained as before after summing over the index $i$.

\subsection{A special case: Poisson arrivals with exponential demand size}

Now, we present the expressions for the special case of Poisson demands with rate $\lambda$ and exponential batch sizes with mean $1 / \alpha$. Let $p(k, \lambda)$ and $P(k, \lambda)$ represent, respectively, the p.m.f. and d.f. of a Poisson random variable with rate $\lambda$. Then, for $k=1,2, \ldots$, we have $F_{k}(x)=$ $\bar{P}(k-1, \lambda x), \quad v_{k}(x)=\alpha p(k-1, \alpha x)$ and $V_{k}(x)=\bar{P}(k-$ $1, \alpha x)$. For this special case, the foregoing expressions are written as

$$
\begin{aligned}
& f_{r_{1}}(x) \\
& =\alpha e^{\alpha x} \sum_{k=0}^{\infty} \int_{z=0}^{\infty}[\bar{P}(k-1, \alpha S) p(k, \lambda z) \\
& \left.\quad+e^{\alpha(S-s)} p(k-1, \alpha S) \bar{P}(k-1, \lambda z)\right] \mathrm{d} G(z), \\
& E[C L(s, S)] \\
& =\alpha \sum_{k=0}^{\infty}\left[\bar{P}(k-1, \alpha S) \int_{z=0}^{\infty} \bar{P}(k-1, \lambda z) \mathrm{d} G(z)\right. \\
& \left.\quad+\int_{x=0}^{\infty} \bar{P}(k-1, \alpha(x-s)) \mathrm{d} F_{r_{1}}(x)\right],
\end{aligned}
$$

$$
\begin{aligned}
& E[C L(s, S)] \\
& =\alpha \sum_{k=0}^{\infty}\left[\bar{P}(k-1, \alpha S) \int_{z=0}^{\infty} \bar{P}(k-1, \lambda z) \mathrm{d} G(z)\right. \\
& \left.\quad+\int_{x=0}^{\infty} \bar{P}(k-1, \alpha(x-s)) \mathrm{d} F_{r_{1}}(x)\right], \\
& E[H C(s, S)] \\
& =h \sum_{i=1}^{\infty}\left[S \bar{P}(i-2, \alpha S)-\frac{(i-1)}{\alpha} \times \bar{P}(i-1, \alpha S)\right] \\
& \quad \times\left[\frac{1}{\lambda}-\int_{x=0}^{\infty} p(i-1, \lambda x) G(x) \mathrm{d} x\right], \\
& E[P C(s, S)] \\
& =\pi \sum_{i=1}^{\infty}\left[S \bar{P}(i-2, \alpha S)-\frac{(i-1)}{\alpha} \bar{P}(i-1, \alpha S)\right] \\
& \quad \times \int_{x=0}^{\infty} p(i-1, \lambda x) \mathrm{d} G(x) . \\
& E[U S C(s, S)] \\
& =-b \int_{x=s}^{0}\left[s+\frac{1}{\alpha} \bar{P}(0, \alpha(x-s))\right] \mathrm{d} F_{r_{1}}(x), \\
& E[T D S C(s, S)] \\
& =\rho \alpha \int_{x=s}^{0} \sum_{i=1}^{\infty}\left[\frac{i-1}{\alpha} \bar{P}(i-1, \alpha(x-s))\right. \\
& \quad-x \bar{P}(i-2, \alpha(x-s))] \mathrm{d} F_{r_{1}}(x) .
\end{aligned}
$$

\section{Quasi-convexity of the average cost for unit demands}

The unit demand case is important from both theoretical and practical aspects and is one of the most frequently assumed models in the literature. We provide some structural results for this special case below. If we let $v_{k}(x)=I(x=k)$, the expressions for the operating characteristics simplify as

$$
\begin{aligned}
E[C L(s, S)] & =\mu\left[-s+\sum_{k=1}^{S} \int_{x=0}^{\infty} \bar{G}(x) \mathrm{d} F_{k}(x)\right], \\
E[S C(s, S)] & =b(-s-1)+\rho \mu s(s+1) / 2 \equiv C_{1}(s), \\
E[H C(s, S)] & =h \sum_{k=1}^{S} \int_{0}^{\infty} \bar{G}(x) \overline{F_{k}}(x) \mathrm{d} x \equiv h C_{2}(S), \\
E[P C(s, S)] & =\pi \sum_{k=1}^{S} \int_{0}^{\infty} \overline{F_{k}}(x) \mathrm{d} G(x) \\
& =\pi\left[S-\sum_{k=1}^{S} P\left(\tau>X_{k}\right)\right] \equiv \pi C_{3}(S),
\end{aligned}
$$

where $S C(s, S)$ is the sum of the unit and the timedependent shortage costs in a cycle. We next show that $A C(s, S)$ is quasi-convex in $(s, S)$ for unit demands. Since quasi-convexity implies unimodality, this result is of both theoretical and practical importance. In the literature, 
convexity properties of functions are usually obtained over convex sets which are not readily applicable to our setting due to the discrete structure of the average cost rate function. Hence, we provide general definitions for arbitrary sets below.

Definition 1. Let $\theta$ be a function defined on an arbitrary nonempty set, $\mathcal{X}$. Also, let $\gamma \in[0,1]$ and $x^{*}, x, \bar{x} \in \mathcal{X}$ subject to $\gamma x+(1-\gamma) \bar{x} \in \mathcal{X}$.

(i) $\theta$ is convex at a point (concave at a point) $\bar{x} \in \mathcal{X}$ if

$$
\theta(\gamma x+(1-\gamma) \bar{x}) \leq(\geq) \gamma \theta(x)+(1-\gamma) \theta(\bar{x}) .
$$

(ii) $\theta$ is convex (concave) on $\mathcal{X}$ if $\theta$ is convex (concave) at every point $x \in \mathcal{X}$.

(iii) $\theta$ is quasi-convex at a point $\bar{x} \in \mathcal{X}$ if

$$
\theta(\gamma x+(1-\gamma) \bar{x}) \leq \max [\theta(x), \theta(\bar{x})] .
$$

(iv) $\theta$ is quasi-convex in $\mathcal{X}$ if $\theta$ is quasi-convex at every point $x \in \mathcal{X}$.

(v) $x^{*} \in \mathcal{X}$ is a local minimum of $\theta$ if and only if there exists a subset $A=\left\{x:\left|x-x^{*}\right| \leq \epsilon, x \neq x^{*} \epsilon>0\right\} \subset$ $\mathcal{X}$ such that $\forall x \in A, \theta\left(x^{*}\right) \leq \theta(x)$.

(vi) If $x^{*}$ is unique in $A \subset \mathcal{X}$, then it is a strict local minimum.

The following two lemmas generalize the results of Avriel (1976, p. 156) on the analytical properties of non-linear fractional functions defined at arbitrary non-empty sets for which the proof is given only for the latter since the former is straightforward.

Lemma 3. Let $\varphi_{1}(x)$ and $\varphi_{2}(x)$ be real-valued functions defined on an arbitrary set, $\mathcal{X}$. Let $\varphi_{1}(x)$ be a non-negative and convex and $\varphi_{2}(x)$ be a positive and concave function on $\mathcal{X}$. Then $\varphi(x)=\varphi_{1}(x) / \varphi_{2}(x)$ is a quasi-convex function on $\mathcal{X}$.

Lemma 4. Let $\mathcal{X}$ be an arbitrary set and $\varphi$ be a real-valued quasi-convex function on $\mathcal{X}$ and $x^{*} \in \mathcal{X}$ be a strict local minimum of $\varphi$ on $\mathcal{X}$. Then $x^{*}$ is a strict global minimum.

Proof. See the Appendix.

Next we have some results regarding the convexity behavior of the expected cycle length and cycle cost functions.

\section{Lemma 5.}

(i) $E[C L(s, S)]$ is concave in $(s, S)$.

(ii) $E[C C(s, S)]$ is convex in $(s, S)$.

Proof. See the Appendix.

We next have the following theorem which states a strong analytical property of the cost rate function which follows directly from Lemma 3 and Lemma 5.

Theorem 1. $A C(s, S)$ is quasi-convex in $(s, S)$.

We finally have the following theorem, the proof of which follows from Lemma 4 and Theorem 1.

Theorem 2. A strict local minimum, $\left(s^{*}, S^{*}\right)$ is also a strict global minimum of the average cost rate function, $A C(s, S)$.

\section{A heuristic for positive lead times}

In the previous sections, we considered the analysis of the $(s, S)$ policy for perishable goods with zero lead time. When a positive lead time is introduced, the model becomes highly complicated since one has to keep track of the remaining lead times and the remaining shelf lives of different batches in the system at a given instant. As another issue, when $L=$ 0 , the First-In First-Out (FIFO) issuing policy is optimal and in fact two batches do not exist in stock simultaneously since $s<0$. When $L>0$ and the shelf life is fixed, the FIFO policy would still be optimal since it is implicitly assumed that the items are sold at the same price. However, when the shelf life is random and $L>0$, FIFO would no longer be optimal since a batch that arrives later may have a shorter shelf life, in which case a policy where items with shorter shelf lives are sold first should be employed. Such a policy would unfortunately make the analysis intractible and we therefore assume a FIFO policy also for $L>0$.

For a fixed shelf life and positive lead times, Lian and Liu (2001) proposed a heuristic based on the optimal and order-up-to levels $\left(s_{0}, S_{0}\right)$ of the model with $L=0$. They use the modified reorder and order-up-to levels $\left(s_{1}, S_{1}\right)$ given as $s_{1}=s_{0}+D_{\mathrm{L}}+E P$ and $S_{1}=S_{0}+D_{\mathrm{L}}+E P$ where $D_{\mathrm{L}}$ is the expected demand during the lead time and $E P$ is the expected number of items that perish during a cycle. As confirmed with our experiments, the results presented by Lian and Liu (2001) show that this heuristic overestimates the reorder and order-up-to levels by adding an inflated quantity $E P$. We believe that a more effective heuristic should make use of the information about the remaining shelf life of the products in stock at the time of order placement for the estimation of the number of units expected to perish during a lead time. For our heristic, we assume that the batch size is constant given by $\beta$, the expected batch size. The demand during the lead time is estimated by $D_{\mathrm{L}}=\lceil L \beta / \mu\rceil$, where $\lceil x\rceil$ is the smallest integer greater than or equal to $x$, and the inventory level at the time of order placement is estimated by

$$
\tilde{s}_{0}=S_{0}-\left\lceil\left(S_{0}-s_{0}\right) / \beta\right\rceil \beta .
$$

We then adjust the order-up-to and reorder levels as $S_{1}=$ $S_{0}+D_{\mathrm{L}}$ and $s_{1}=\tilde{s}_{0}+D_{\mathrm{L}}$. If $s_{1} \leq 0$, the on-hand inventory is zero at the beginning of the lead time and we propose to use $\left(s_{1}, S_{1}\right)$ as our heuristic, since no units perish during the lead time. Otherwise, since the items in stock at the instance of order placement are at risk of degrading during the lead time, we estimate the expected number of degraded units and further adjust $S_{1}$. To describe the estimation method, let us define $k_{1}$ and $k_{2}$ as the estimated number of demand arrivals that makes the inventory position drop from $S_{1}$ to or below $s_{1}$ and from $s_{1}$ to or below zero respectively. Also let $\tau_{\mathrm{r}}=\tau-X_{k_{1}}$ denote the remaining shelf life of the batch in use at the time of order placement. Let $p=F_{k_{1}}(\tau)-$ $F_{k_{1}}(\tau-L)$ denote the probability that degradation occurs during the lead time and given that degradation occurs, we 
approximate the number of perished units as

$$
P_{\mathrm{r}}=\sum_{i=1}^{k_{2}}\left(s_{1}-(i-1) \beta\right) I\left(X_{i-1}<\tau_{\mathrm{r}}<X_{i}\right)
$$

with expected value

$$
\begin{aligned}
E\left[P_{\mathrm{r}}\right]= & s_{1}\left(F_{k_{1}}(\tau)-F_{k_{1}+k_{2}}(\tau)\right) \\
& +k_{2} \beta F_{k_{1}+k_{2}}(\tau)-\beta \sum_{i=1}^{k_{2}} F_{k_{1}+i}(\tau)
\end{aligned}
$$

Then, the approximate number of units that perish during the lead time is taken as $E\left[P_{\mathrm{L}}\right]=p E\left[P_{\mathrm{r}}\right]$.

We summarize our proposed heuristic in the following steps:

Step 1. Calculate $\left(s_{0}, S_{0}\right)$ of the model with $L=0$ and referring to Equation (24), let $\left(s_{1}, S_{1}\right)=\left(\tilde{s}_{0}+D_{\mathrm{L}}, S_{0}+\right.$ $\left.D_{\mathrm{L}}\right)$.

Step 2. If $s_{1} \leq 0,\left(s_{1}, S_{1}\right)$ are the proposed optimal parameters.

Step 3. If $s_{1}>0$, set $k_{1}=\left\lceil\left(S_{1}-s_{1}\right) / \beta\right\rceil$ and $k_{2}=\left\lceil s_{1} / \beta\right\rceil$. The suggested optimal parameters are $\left(s_{1}, S_{1}+\right.$ $\left.\left\lceil E\left[P_{\mathrm{L}}\right]\right\rceil\right)$.

In the heuristic proposed above, $\tau$ can be fixed or random. If it is random, then expectations of $E\left[P_{\mathrm{r}}\right]$ and $p$ should be taken with respect to $\tau$. Note also that the proposed heuristic is applicable to both discrete and continuous demand batches. The performance of the heuristic proposed above is compared to that of Lian and Liu (2001) in the next section.

\section{Numerical results}

We conducted an extensive numerical study to address several issues regarding the inventory control of perishable items with a fixed and a random shelf life. Major issues considered are the sensitivity of the optimal policy to different choices of system parameters, the loss due to ignorance of the randomness of the shelf life, the impact of the shape of the shelf life distribution and finally the performance of the proposed heuristic for positive lead times. To elaborate on these, various cases of cost parameters, batch distributions, the mean $\mu_{\tau}$, the coefficient of variation (c.o.v.), $C_{\tau}$, distributions of the shelf life $\tau$ and c.o.v. $C_{X}$ of $X$, are investigated. For the batch distribution, unit $\left(B D_{1}\right)$, geometric $\left(B D_{2}\right)$ and gamma $\left(B D_{3}\right)$ demand batches with mean $\beta=5$ and variance 20 are considered. The comparisons for different batches are based on fixed values of the average number of units demanded per time, denoted with $\alpha=\beta / \mu$. We considered both exponential and 4-Erlang inter-arrival times, the parameters of which are selected to match the assumed $\alpha$ values. For example, if $\alpha=25$, with a geometric or gamma batch with mean $\beta=5$, the mean inter-demand time is $\mu=0.2$ and the parameters of the exponential and
Erlang distributions are five and 20, respectively. Also, to standardize the selection of the experimental values of the mean shelf life, we consider the average time required to consume the modified (since we have batch demands) order quantity $Q=\sqrt{2 K \alpha / h}$ of the economic order quality model. That is, we set $T=Q / \alpha$ and the values of $E[\tau]$ are selected as multiples of $T$. Throughout our experiments with a zero lead time, the holding cost $h=1$ and the ordering cost $K=50$ are used. Six different shelf life distributions are considered to investigate factors such as the shape, skewness, tail probabilities and the range of the shelf life distribution. These distributions are respectively the gamma, Weibull, uniform, triangular, and left-truncated gamma with two different truncation values. Gamma and Weibull distributions are commonly used to represent time to failure, hence are considered here for the shelf life. The uniform distribution is interesting from a practical point of view since it represents a "non-informative" case and the triangular distribution is a three-parameter distribution which may be used as a simple approximation to more complicated ones. Finally, truncated gamma distributions are included to cover the cases where there may be a positive threshold for the shelf life below which products may not be acceptable. The two truncation points are taken as the lower limits of the uniform and triangular distributions respectively. The parameters of all the distributions are adjusted to match the selected mean and the c.o.v. of the shelf life.

\subsection{Sensitivity analysis}

We start with discussing the sensitivity of the optimal policy parameters and the cost rate to system parameters. In the tables presenting the numerical results, optimal policy parameters and optimal cost rate are denoted by $\left(s_{0}, S_{0}\right)$ and $A C^{*}$ respectively.

Table 1 provides a representative set of our results. The shelf life is gamma with $\alpha=25$ and $C_{X}=0.5$. We observe that the results are in agreement with expectations, in that both $s_{0}$ and $S_{0}$ decrease in general with the perishing cost. When the shelf life variability is high, the order-upto levels become more sensitive to changes in the perishing costs. For example, when $C_{\tau}=1$, we observe a more drastic decrease in $S_{0}$ as $\pi$ increases from three to 15 , than the corresponding decrease when $C_{\tau}=0.5$. When the perishing cost is large, the policy parameters become highly sensitive to both the mean and the variability of the shelf life. Consider for example the case with geometric demand $\left(B D_{2}\right), C_{\tau}=1.00, b=6, \rho=2$ and $\pi=15$. We observe that when $E[\tau]$ drops from $1.50 T$ to $1.00 T$ the change in $S_{0}$ is $27 \%$ (from 15 to 11 ) and when $E[\tau]$ drops from $1.00 T$ to $0.75 T$, it is $18 \%$ (from 11 to nine). Note, however, that the corresponding decreases are significantly lower, $14 \%$ (from 28 to 24 ) and 13\% (from 24 to 21 ) for $\pi=3$. 
Table 1. Sensitivity analysis with gamma shelf life and $\alpha=25-$ gamma inter-arrival time with c.o.v $=0.50$

\begin{tabular}{|c|c|c|c|c|c|c|c|c|c|c|c|c|c|c|c|}
\hline \multirow{2}{*}{\multicolumn{4}{|c|}{ Batch distribution }} & \multicolumn{6}{|c|}{$C_{\tau}=0.50$} & \multicolumn{6}{|c|}{$C_{\tau}=1.00$} \\
\hline & & & & \multicolumn{2}{|c|}{$B D_{1}$} & \multicolumn{2}{|c|}{$B D_{2}$} & \multicolumn{2}{|l|}{$B D_{3}$} & \multicolumn{2}{|c|}{$B D_{1}$} & \multicolumn{2}{|c|}{$B D_{2}$} & \multicolumn{2}{|l|}{$B D_{3}$} \\
\hline$E[\tau] / T$ & $\pi$ & $b$ & $\rho$ & $\left(s_{0}, S_{0}\right)$ & $A C^{*}$ & $\left(s_{0}, S_{0}\right)$ & $A C^{*}$ & $\left(s_{0}, S_{0}\right)$ & $A C^{*}$ & $\left(s_{0}, S_{0}\right)$ & $A C^{*}$ & $\left(s_{0}, S_{0}\right)$ & $A C^{*}$ & $\left(s_{0}, S_{0}\right)$ & $A C^{*}$ \\
\hline \multirow[t]{8}{*}{0.75} & \multirow[t]{4}{*}{3} & \multirow[t]{2}{*}{2} & 2 & $(-10,26)$ & 68.35 & $(-10,23)$ & 62.39 & $(-10.3,22.7)$ & 63.01 & $(-19,21)$ & 87.09 & $(-15,18)$ & 76.97 & $(-15.3,18.3)$ & 77.63 \\
\hline & & & 4 & $(-5,27)$ & 69.81 & $(-7,23)$ & 64.24 & $(-7.3,22.7)$ & 64.72 & $(-11,23)$ & 93.46 & $(-10,19)$ & 81.48 & $(-10.6,19.0)$ & 82.09 \\
\hline & & \multirow[t]{2}{*}{6} & 2 & $(-1,27)$ & 71.37 & $(-3,24)$ & 68.32 & $(-2.7,24.3)$ & 68.39 & $(-1,25)$ & 105.16 & $(-4,21)$ & 90.61 & $(-4.3,21.0)$ & 91.26 \\
\hline & & & 4 & $(-1,27)$ & 71.37 & $(-3,24)$ & 68.54 & $(-3.0,24.0)$ & 69.56 & $(-1,25)$ & 105.16 & $(-4,21)$ & 91.14 & $(-4,21.3)$ & 91.77 \\
\hline & 15 & 2 & 2 & $(-15,18)$ & 78.62 & $(-13,15)$ & 71.65 & $(-13.3,15.3)$ & 73.34 & $(-28,9)$ & 104.19 & $(-23,6)$ & 93.92 & $(-23 \cdot 0,5.7)$ & 94.02 \\
\hline & & & 4 & $(-9,19)$ & 82.53 & $(-9,15)$ & 75.16 & $(-9.3,15.0)$ & 76.10 & $(-18,10)$ & 119.44 & $(-15,7)$ & 105.06 & $(-14.7,7.3)$ & 105.79 \\
\hline & & 6 & 2 & $(-1,19)$ & 88.60 & $(-4,16)$ & 82.68 & $(-3.7,15.7)$ & 84.10 & $(-9,13)$ & 166.81 & $(-7,9)$ & 128.38 & $(-7.3,9.3)$ & 129.01 \\
\hline & & & 4 & $(-1,19)$ & 88.60 & $(-3,16)$ & 83.12 & $(-3.0,16.3)$ & 83.90 & $(-5,13)$ & 169.03 & $(-6,9)$ & 130.46 & $(-6.0,9.3)$ & 131.08 \\
\hline \multirow[t]{8}{*}{1.00} & \multirow[t]{4}{*}{3} & \multirow[t]{2}{*}{2} & 2 & $(-7,31)$ & 62.13 & $(-8,27)$ & 57.47 & $(-8.3,27.3)$ & 58.11 & $(-16,25)$ & 81.74 & $(-13,21)$ & 72.37 & $(-13.3,21.0)$ & 73.02 \\
\hline & & & 4 & $(-4,31)$ & 62.69 & $(-6,27)$ & 58.71 & $(-6.3,27.0)$ & 59.18 & $(-10,26)$ & 86.11 & $(-9,22)$ & 75.68 & $(-9.0,22.0)$ & 76.25 \\
\hline & & \multirow[t]{2}{*}{6} & 2 & $(-1,31)$ & 63.16 & $(-3,28)$ & 61.55 & $(-2.7,28.3)$ & 63.16 & $(-1,28)$ & 92.83 & $(-4,24)$ & 82.46 & $(-3.7,24.3)$ & 82.97 \\
\hline & & & 4 & $(-1,31)$ & 63.16 & $(-2,28)$ & 61.73 & $(-2.3,27.7)$ & & $(-1,28)$ & 92.83 & $(-3,24)$ & 82.84 & $(-3.7,24.0)$ & 83.29 \\
\hline & \multirow[t]{4}{*}{15} & 2 & 2 & $(-11,23)$ & 71.46 & $(-11,19)$ & 65.48 & $(-11.0,19.3)$ & 66.14 & $(-26,11)$ & 100.69 & $(-21,9)$ & 90.27 & $(-21.3,9.3)$ & 91.05 \\
\hline & & & 4 & $(-6,23)$ & 73.49 & $(-8,19)$ & 67.79 & $(-8.3,19.0)$ & 67.46 & $(-16,12)$ & 113.58 & $(-14,10)$ & 99.43 & $(-14.0,9.7)$ & 99.89 \\
\hline & & 6 & 2 & $(-1,23)$ & 75.97 & $(-3,20)$ & 72.75 & $(-3.3,20.3)$ & 73.48 & $(-1,15)$ & 150.39 & $(-6,11)$ & 117.97 & $(-6.0,10.4)$ & 118.49 \\
\hline & & & 4 & $(-1,23)$ & 75.97 & $(-3,20)$ & 72.98 & $(-3.0,20.3)$ & 72.71 & $(-1,15)$ & 150.39 & $(-5,12)$ & 119.42 & $(-5.3,12.3)$ & 119.77 \\
\hline \multirow[t]{8}{*}{1.50} & \multirow[t]{4}{*}{3} & \multirow[t]{2}{*}{2} & 2 & $(-3,38)$ & 55.61 & $(-7,33)$ & 52.38 & $(-6.7,33.3)$ & 52.90 & $(-13,30)$ & 74.45 & $(-11,26)$ & 66.36 & $(-11.3,26.0)$ & 67.02 \\
\hline & & & 4 & $(-2,38)$ & 55.71 & $(-5,33)$ & 53.18 & $(-5.3,33.3)$ & 52.71 & $(-7,31)$ & 76.79 & $(-8,26)$ & 68.54 & $(-8.3,26.0)$ & 69.11 \\
\hline & & \multirow[t]{2}{*}{6} & 2 & $(-1,38)$ & 55.75 & $(-2,34)$ & 55.04 & $(-2.3,34.3)$ & 55.59 & $(-1,32)$ & 79.69 & $(-3,28)$ & 73.03 & $(-3.3,28.3)$ & 73.81 \\
\hline & & & 4 & $(-1,38)$ & 55.75 & $(-2,34)$ & 55.09 & $(-2.3,34.3)$ & 54.64 & $(-1,32)$ & 79.69 & $(-3,28)$ & 73.24 & $(-3.0,28.3)$ & 74.03 \\
\hline & \multirow[t]{3}{*}{5} & 2 & 2 & $(-7,30)$ & 62.32 & $(-9,25)$ & 57.91 & $(-9.3,25.3)$ & 57.48 & $(-23,15)$ & 94.91 & $(-18,12)$ & 84.38 & $(-18.3,12.3)$ & 85.08 \\
\hline & & & 4 & $(-4,30)$ & 62.89 & $(-6,25)$ & 59.18 & $(-5.7,25.3)$ & 59.77 & $(-14,16)$ & 104.37 & $(-12,13)$ & 90.96 & $(-12.0,13.3)$ & 91.67 \\
\hline & & 6 & 2 & $(-1,30)$ & 63.37 & $(-3,26)$ & 62.08 & $(-3.3,26.3)$ & 62.70 & $(-1,19)$ & 125.42 & $(-5,15)$ & 103.88 & $(-5.6,15.6)$ & 104.55 \\
\hline & & & 4 & $(-1,30)$ & 63.37 & $(-3,26)$ & 62.27 & $(-3.0,26.0)$ & 61.89 & $(-1,19)$ & 125.42 & $(-5,15)$ & 104.79 & $(-5.3,15.3)$ & 105.46 \\
\hline
\end{tabular}

As to the batch size, geometric and gamma demands yield smaller $S_{0}$ values than the unit demand. When the backorder costs are small, smaller $s_{0}$ values are observed for the unit demand, whereas the reverse is usually true with higher backorder costs. The optimal policy parameters and the cost rate of geometric and gamma demands are less sensitive to the cost and model parameters. Overall, the optimal cost rates for geometric and gamma demands are much smaller than that of the unit demand. We also observe that the geometric and gamma demands with the same variance yield very similar results in terms of the optimal cost and the policy parameters.

The effect of the c.o.v. of the inter-demand time $\left(C_{X}\right)$ has a small effect on both $\left(s_{0}, S_{0}\right)$ and $A C^{*}$. In the unreported results for $C_{X}=1$, which corresponds to Poisson arrivals, we observed that the optimal policy parameters changed in only four of the 48 instances with unit demand, 29 of the 42 with geometric and gamma demands. In these instances, the exponential demand usually results in lower $s_{0}$ and/or $S_{0}$ values with a maximum change of only one unit. With unit demand and $C_{\tau}=0.5$, the $A C^{*}$ of $C_{X}=0.5$ is always smaller than that of $C_{X}=1$. For $C_{\tau}=1$, exponential arrivals resulted in slightly lower cost rates with an average difference of $0.29 \%$. We also observed similar behavior with geometric and gamma demands except that the average differences were higher. For $C_{\tau}=0.5$, the average differences between the cases of $C_{X}=0.5$ and $C_{X}=1$ are 2.73 and $2.81 \%$ for geometric and gamma demands, respectively, whereas with $C_{\tau}=1$, the corresponding figures are 1.25 and $1.27 \%$.

\subsection{The impact of constant versus random shelf life}

Next, we discuss whether incorporating the randomness of the shelf life is crucial and if its distribution has a notable impact on the costs. In the following, the model that considers the shelf life as a random variable is referred to as the random model and the model that treats the shelf life as fixed (at the mean of the random shelf life) is referred to as the constant (or fixed) model. Let $\left(s_{0}, S_{0}\right)$ and $\left(s_{\mathrm{c}}, S_{\mathrm{c}}\right)$ be the optimal policy parameters of the random and the constant models respectively and let $A C(s, S)$ denote the average cost rate of the random model evaluated at $(s, S)$ with $A C^{*}$ being the optimal one. As a measure of the loss due to the ignorance of the randomness of the shelf life, we consider $\Delta_{0} \%$ below.

$$
\Delta_{0} \%=\frac{A C\left(s_{\mathrm{c}}, S_{\mathrm{c}}\right)-A C\left(s_{0}, S_{0}\right)}{A C\left(s_{0}, S_{0}\right)} \times 100 .
$$

A representative set of our results with $C_{\tau}=0.50$ and $\alpha=50$ for the six shelf life distributions presented before are given in Tables 2 and 3 for the unit and gamma demands. We exclude geometric demand here since it 


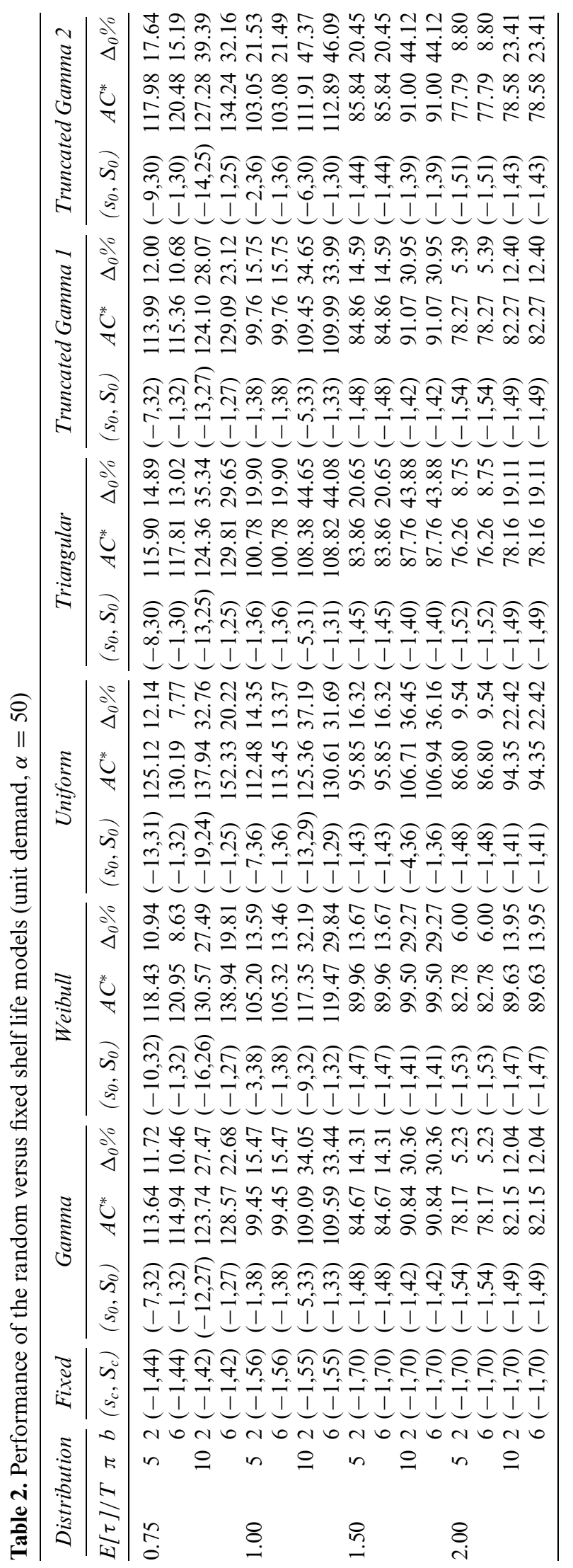




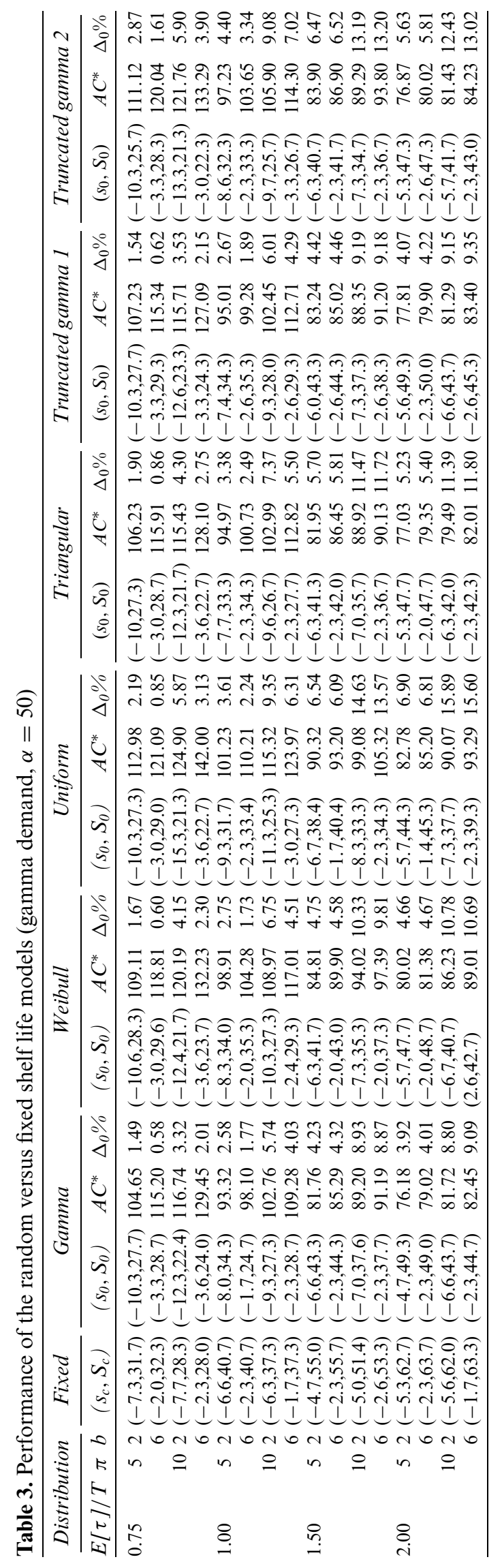


provides results similar to gamma demand as presented in Table 1.

One of the main conclusions from the results of Tables 2 and 3 is that explicitly modeling the randomness of the shelf life makes significant differences in the cost rates, especially for unit demands for which $\Delta_{0} \%$ can be as large as $34 \%$, which is $44 \%$ for the gamma and triangular distributions. The batch demand seems to provide some robustness, however, even in this case the loss due to ignorance of randomness can be as large as 9 and $11 \%$ for the same distributions. The results also indicate that the shape of the shelf life distribution is quite effective on optimal policy parameters, cost rate and the $\Delta_{0} \%$ values. Usually, gamma distribution yields the smallest and the uniform distribution yields the highest cost rate and $\Delta_{0} \%$ values. The results for the triangular distribution are very similar to those for the gamma distribution, which suggests for this problem that it can be used as a simple approximation to the gamma distribution. When we compare the gamma and the truncated gamma distributions, we see that the costs increase as the left threshold becomes larger, which might seem counterintuitive at first sight. This results from forcing the mean of all three distributions to be the same and as the lower threshold increases, the smaller values close to the threshold attain more probability in order to keep the mean constant. This observation brings about an interesting point: if the truncation value (lower threshold) represents a lower limit on the shelf life that the retailer is willing to impose in a contract with a supplier, the retailer should be careful to pay more attention to the distribution of the shelf lives rather than the threshold value.

Table 4 presents the percentage difference between the maximum and minimum costs among the six shelf life distributions. We again observe big differences among the costs with respect to different shelf life distributions and costs almost double for the unit demand compared to geometric demand. For gamma demand, the percentage difference is between those two. Hence, improving the storage conditions or the production process in general that would result in longer-tailed shelf life distributions may result in significant savings. We also investigated the impact of the variability of the shelf life and considered the cases for $C_{\tau}=0.75,1.0$. From the results unreported herein, we observed that as $C_{\tau}$ increases the difference between the constant and the random model increases considerably and the highest costs are incurred for the exponential shelf life. Since an exponential distribution is commonly used for modeling shelf life, our results indicate a warning about its usage unless there is a strong empirical evidence.

\subsection{Performance of the proposed heuristic for positive lead times}

In this section we present the performance of the proposed heuristic, $\mathrm{H}_{1}$, over a wide range of parameters for a random shelf life and compare the performance of it to that of a recently suggested one, $\mathrm{H}_{2}$, by Lian and Liu (2001) for a fixed shelf life.

For measuring the performance of the heuristics, a simulation model is used. One simulation run is obtained by generating 10000 time units in the $(s, S)$ model, from which the average cost rate is obtained. For a given $(s, S)$ pair, the final cost rate is obtained by taking the average of ten simulation runs, over which the optimal values of $(s, S)$ are searched. The following performance measure, $\Delta_{\mathrm{L}} \%$, for both heuristics is considered:

$$
\Delta_{\mathrm{L}} \%=\frac{A C_{\mathrm{L}}\left(s_{i}^{*}, S_{i}^{*}\right)-A C_{\mathrm{L}}\left(s^{*}, S^{*}\right)}{A C_{\mathrm{L}}\left(s^{*}, S^{*}\right)} \times 100 .
$$

where for $i=1,2,\left(s_{i}^{*}, S_{i}^{*}\right)$ and $\left(s^{*}, S^{*}\right)$ are the optimal policy parameters obtained from heuristic $\mathrm{H}_{i}$ and the simulation model respectively and $A C_{\mathrm{L}}$ is the estimated average cost calculated from simulation.

The results for the proposed heuristic are given in Table 5 for unit demand and $\pi=5, b=2, \rho=2$. We also provide underneath each distribution, the average deviation of the heuristic from the simulation in the presented 12 cases, as well as the corresponding average for the batch demand case, for which we have not provided details to save space. We observe that the performance of $\mathrm{H}_{1}$ is in general very good for all distributions, with an average of less than $1 \%$ for unit demands and less than $2 \%$ in batch demands. We also note that its performance is comparable among various distributions and the batch size has more impact than the shelf life distribution. The relatively poor performance for batch demands may result from the fact that the proposed

Table 4. Percentage cost differences across shelf life distributions

\begin{tabular}{|c|c|c|c|c|c|c|c|c|c|c|c|c|c|c|c|c|}
\hline \multirow{4}{*}{$\begin{array}{l}\pi \\
b\end{array}$} & \multicolumn{16}{|c|}{$E[\tau] / T$} \\
\hline & \multicolumn{4}{|c|}{0.75} & \multicolumn{4}{|c|}{1.00} & \multicolumn{4}{|c|}{1.50} & \multicolumn{4}{|c|}{2.00} \\
\hline & \multicolumn{2}{|c|}{5} & \multicolumn{2}{|c|}{10} & \multicolumn{2}{|c|}{5} & \multicolumn{2}{|c|}{10} & \multicolumn{2}{|c|}{5} & \multicolumn{2}{|c|}{10} & \multicolumn{2}{|c|}{5} & \multicolumn{2}{|c|}{10} \\
\hline & 2 & 6 & 2 & 6 & 2 & 6 & 2 & 6 & 2 & 6 & 2 & 6 & 2 & 6 & 2 & 6 \\
\hline$B D_{1}$ & 10.10 & 13.27 & 11.48 & 18.48 & 13.10 & 14.08 & 15.67 & 20.02 & 14.30 & 14.30 & 21.59 & 21.86 & 13.82 & 13.82 & 20.71 & 20.71 \\
\hline$B D_{2}$ & 5.90 & 7.07 & 7.27 & 9.84 & 7.37 & 8.57 & 9.28 & 11.34 & 8.30 & 9.20 & 11.40 & 12.97 & 8.40 & 9.05 & 12.26 & 13.40 \\
\hline$B D_{3}$ & 6.96 & 6.11 & 7.48 & 10.73 & 7.19 & 9.34 & 10.56 & 12.11 & 9.47 & 9.65 & 12.14 & 13.20 & 8.66 & 9.82 & 12.71 & 13.80 \\
\hline
\end{tabular}




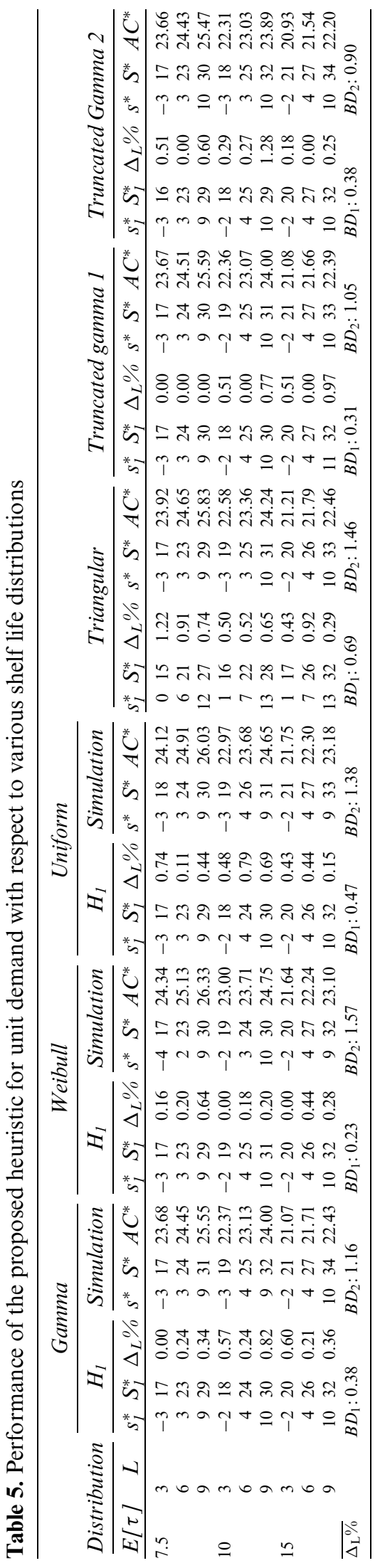


heuristic does not explicitly take into account the batch size distribution.

Next we want to discuss the performance of the proposed heuristic relative to an existing one, namely the one by Lian and Liu (2001) proposed for a fixed shelf life. Ta- ble 6 presents a representative set of our results for a fixed shelf life with $\rho=2$. We observe that $\mathrm{H}_{1}$ performs better than $\mathrm{H}_{2}$ in most cases. In particular, note that for the geometric demand, $\mathrm{H}_{1}$ is uniformly better than $\mathrm{H}_{2}$. This may be explained by the overestimation of the reorder levels by

Table 6. Performance of the proposed and available heuristics for fixed shelf life

\begin{tabular}{|c|c|c|c|c|c|c|c|c|c|c|c|c|c|c|c|c|c|c|c|c|c|}
\hline \multirow[b]{3}{*}{$E[\tau]$} & \multirow[b]{3}{*}{$L$} & \multirow[b]{3}{*}{$\pi$} & \multirow[b]{3}{*}{$b$} & \multicolumn{9}{|c|}{$B D_{1}$} & \multicolumn{9}{|c|}{$B D_{2}$} \\
\hline & & & & \multicolumn{3}{|c|}{$H_{1}$} & \multicolumn{3}{|c|}{$\mathrm{H}_{2}$} & \multicolumn{3}{|c|}{ Simulation } & \multicolumn{3}{|c|}{$H_{1}$} & \multicolumn{3}{|c|}{$H_{2}$} & \multicolumn{3}{|c|}{ Simulation } \\
\hline & & & & $s_{1}^{*}$ & $S_{1}^{*}$ & $\Delta_{L} \%$ & $s_{2}^{*}$ & $S_{2}^{*}$ & $\Delta_{L} \%$ & $s^{*}$ & $S^{*}$ & $A C^{*}$ & $s_{1}^{*}$ & $S_{1}^{*}$ & $\Delta_{L} \%$ & $s_{2}^{*}$ & $S_{2}^{*}$ & $\Delta_{L} \%$ & $s^{*}$ & $S^{*}$ & $A C^{*}$ \\
\hline \multirow[t]{12}{*}{7.5} & 3 & 5 & 2 & -2 & 18 & 0.00 & -2 & 18 & 0.00 & -2 & 18 & 21.85 & -6 & 14 & 0.56 & -2 & 14 & 2.99 & -4 & 17 & 27.03 \\
\hline & & & 6 & 1 & 20 & 0.48 & 2 & 20 & 0.82 & 0 & 19 & 25.20 & -1 & 14 & 3.01 & 0 & 14 & 7.40 & -4 & 18 & 32.48 \\
\hline & & 10 & 2 & -2 & 18 & 0.04 & -2 & 18 & 0.04 & -3 & 18 & 22.21 & -3 & 12 & 2.27 & -2 & 12 & 3.74 & -6 & 14 & 28.27 \\
\hline & & & 6 & 1 & 19 & 0.37 & 2 & 19 & 0.69 & -1 & 18 & 25.75 & -2 & 13 & 2.33 & 0 & 13 & 7.98 & -4 & 16 & 34.12 \\
\hline & 6 & 5 & 2 & 4 & 25 & 0.00 & 5 & 25 & 0.05 & 4 & 25 & 22.67 & 0 & 20 & 1.53 & 5 & 21 & 3.65 & -1 & 23 & 31.06 \\
\hline & & & 6 & 7 & 26 & 0.00 & 8 & 26 & 0.37 & 7 & 26 & 26.15 & 5 & 21 & 2.61 & 7 & 21 & 3.51 & 5 & 24 & 36.54 \\
\hline & & 10 & 2 & 4 & 25 & 0.51 & 5 & 25 & 0.52 & 4 & 24 & 23.10 & 3 & 19 & 3.02 & 5 & 19 & 5.18 & -2 & 19 & 32.68 \\
\hline & & & 6 & 7 & 25 & 0.00 & 8 & 25 & 0.56 & 7 & 25 & 26.76 & 4 & 20 & 1.45 & 7 & 20 & 4.59 & -1 & 23 & 38.77 \\
\hline & 9 & 5 & 2 & 10 & 31 & 0.31 & 11 & 31 & 0.00 & 11 & 31 & 23.29 & 6 & 27 & 0.45 & 11 & 27 & 3.58 & 9 & 29 & 34.07 \\
\hline & & & 6 & 13 & 32 & 0.61 & 14 & 32 & 0.00 & 14 & 32 & 26.61 & 11 & 29 & 1.86 & 13 & 27 & 4.45 & 11 & 31 & 39.53 \\
\hline & & 10 & 2 & 10 & 31 & 0.62 & 11 & 31 & 0.35 & 10 & 30 & 23.78 & 9 & 26 & 1.85 & 11 & 25 & 4.53 & 6 & 27 & 36.07 \\
\hline & & & 6 & 13 & 31 & 0.00 & 14 & 31 & 0.04 & 13 & 31 & 27.38 & 10 & 27 & 1.16 & 13 & 26 & 5.92 & 9 & 29 & 41.88 \\
\hline 10 & 3 & 5 & 2 & -1 & 21 & 0.28 & -1 & 21 & 0.28 & -2 & 21 & 20.63 & -5 & 15 & 1.07 & -2 & 15 & 1.66 & -4 & 17 & 25.66 \\
\hline & & & 6 & 2 & 22 & 0.12 & 2 & 21 & 0.26 & 1 & 21 & 23.34 & -4 & 16 & 1.49 & 0 & 16 & 4.11 & -2 & 18 & 30.55 \\
\hline & & 10 & 2 & -1 & 20 & 0.22 & -1 & 20 & 0.22 & -2 & 20 & 20.79 & -6 & 14 & 0.90 & -2 & 14 & 1.95 & -4 & 15 & 26.58 \\
\hline & & & 6 & 2 & 22 & 0.51 & 2 & 21 & 0.29 & 1 & 21 & 23.55 & 0 & 15 & 3.92 & 0 & 15 & 3.92 & -2 & 18 & 31.76 \\
\hline & 6 & 5 & 2 & 5 & 28 & 0.30 & 5 & 27 & 0.14 & 4 & 27 & 21.28 & 1 & 22 & 0.43 & 5 & 22 & 3.36 & 0 & 25 & 29.26 \\
\hline & & & 6 & 8 & 29 & 0.40 & 8 & 27 & 0.77 & 8 & 28 & 24.03 & 2 & 23 & 0.73 & 7 & 23 & 3.97 & 3 & 24 & 34.05 \\
\hline & & 10 & 2 & 5 & 27 & 0.42 & 5 & 26 & 0.00 & 5 & 26 & 21.46 & 0 & 20 & 0.11 & 5 & 21 & 4.12 & 1 & 21 & 30.43 \\
\hline & & & 6 & 8 & 29 & 0.85 & 8 & 27 & 0.56 & 7 & 27 & 24.34 & 6 & 22 & 3.37 & 7 & 22 & 4.13 & 3 & 23 & 35.47 \\
\hline & 9 & 5 & 2 & 11 & 35 & 0.60 & 11 & 33 & 0.00 & 11 & 33 & 21.82 & 7 & 28 & 0.97 & 11 & 28 & 3.21 & 9 & 30 & 32.12 \\
\hline & & & 6 & 14 & 37 & 1.24 & 14 & 33 & 0.71 & 14 & 35 & 24.57 & 8 & 30 & 2.55 & 13 & 29 & 3.82 & 12 & 33 & 36.98 \\
\hline & & 10 & 2 & 11 & 34 & 0.67 & 11 & 32 & 0.01 & 10 & 32 & 22.06 & 6 & 27 & 0.30 & 11 & 27 & 3.29 & 8 & 28 & 33.63 \\
\hline & & & 6 & 14 & 37 & 2.40 & 14 & 33 & 0.38 & 14 & 34 & 24.96 & 12 & 31 & 1.57 & 13 & 28 & 4.37 & 10 & 30 & 38.71 \\
\hline 15 & 3 & 5 & 2 & -1 & 23 & 0.13 & -1 & 23 & 0.13 & -2 & 23 & 20.01 & -3 & 17 & 0.00 & -1 & 17 & 1.61 & -3 & 17 & 24.19 \\
\hline & & & 6 & 2 & 25 & 0.02 & 2 & 24 & 0.17 & 1 & 25 & 22.27 & -3 & 17 & 1.85 & 2 & 18 & 3.56 & -2 & 23 & 28.38 \\
\hline & & 10 & 2 & -1 & 23 & 0.14 & -1 & 23 & 0.14 & -2 & 23 & 20.02 & -4 & 16 & 0.44 & -1 & 16 & 2.23 & -3 & 17 & 24.53 \\
\hline & & & 6 & 2 & 25 & 0.02 & 2 & 24 & 0.11 & 1 & 25 & 22.29 & -3 & 17 & 0.95 & 0 & 17 & 2.36 & -1 & 20 & 28.97 \\
\hline & 6 & 5 & 2 & 5 & 30 & 0.16 & 5 & 29 & 0.31 & 4 & 30 & 20.44 & 3 & 24 & 1.01 & 6 & 24 & 2.97 & 2 & 27 & 27.36 \\
\hline & & & 6 & 8 & 32 & 0.20 & 8 & 30 & 0.43 & 7 & 31 & 22.75 & 3 & 24 & 2.16 & 8 & 24 & 4.10 & 4 & 29 & 31.47 \\
\hline & & 10 & 2 & 5 & 30 & 0.17 & 5 & 29 & 0.27 & 4 & 30 & 20.47 & 2 & 23 & 0.54 & 6 & 23 & 3.30 & 0 & 25 & 28.06 \\
\hline & & & 6 & 8 & 32 & 0.27 & 8 & 30 & 0.38 & 7 & 31 & 22.79 & 3 & 24 & 1.08 & 7 & 24 & 2.59 & 5 & 26 & 32.35 \\
\hline & 9 & 5 & 2 & 11 & 35 & 0.39 & 11 & 35 & 0.18 & 10 & 35 & 20.87 & 9 & 31 & 0.46 & 12 & 30 & 3.36 & 10 & 35 & 30.15 \\
\hline & & & 6 & 14 & 37 & 0.50 & 14 & 36 & 0.63 & 13 & 37 & 23.20 & 9 & 31 & 2.07 & 14 & 30 & 4.28 & 13 & 36 & 34.19 \\
\hline & & 10 & 2 & 11 & 35 & 0.73 & 11 & 35 & 0.20 & 10 & 35 & 20.89 & 8 & 29 & 1.41 & 12 & 29 & 2.89 & 7 & 32 & 30.96 \\
\hline & & & 6 & 14 & 37 & 0.21 & 14 & 36 & 0.57 & 13 & 37 & 23.25 & 9 & 31 & 1.07 & 13 & 30 & 2.06 & 12 & 34 & 35.22 \\
\hline 20 & 3 & 5 & 2 & -1 & 23 & 0.09 & -1 & 23 & 0.09 & -2 & 24 & 19.99 & -3 & 17 & 0.97 & -1 & 17 & 2.14 & -4 & 21 & 23.43 \\
\hline & & & 6 & 2 & 26 & 0.06 & 2 & 25 & 0.01 & 1 & 26 & 22.19 & -2 & 18 & 2.10 & 2 & 19 & 2.71 & -1 & 24 & 27.20 \\
\hline & & 10 & 2 & -1 & 23 & 0.09 & -1 & 23 & 0.09 & -2 & 24 & 19.99 & -3 & 17 & 0.56 & -1 & 17 & 1.76 & -4 & 21 & 23.62 \\
\hline & & & 6 & 2 & 26 & 0.06 & 2 & 25 & 0.00 & 1 & 26 & 22.19 & -2 & 18 & 1.34 & 2 & 19 & 2.15 & -1 & 24 & 27.52 \\
\hline & 6 & 5 & 2 & 5 & 30 & 0.13 & 5 & 29 & 0.34 & 4 & 30 & 20.39 & 3 & 24 & 2.17 & 6 & 24 & 4.16 & 2 & 27 & 26.31 \\
\hline & & & 6 & 8 & 32 & 0.36 & 8 & 31 & 0.37 & 7 & 32 & 22.61 & 4 & 25 & 3.14 & 8 & 25 & 3.73 & 5 & 30 & 30.05 \\
\hline & & 10 & 2 & 5 & 30 & 0.13 & 5 & 29 & 0.34 & 4 & 30 & 20.39 & 3 & 24 & 1.79 & 6 & 24 & 3.82 & 2 & 27 & 26.59 \\
\hline & & & 6 & 8 & 32 & 0.37 & 8 & 31 & 0.37 & 7 & 32 & 22.61 & 4 & 25 & 2.50 & 8 & 25 & 3.22 & 4 & 29 & 30.45 \\
\hline & 9 & 5 & 2 & 11 & 36 & 0.18 & 11 & 35 & 0.43 & 10 & 36 & 20.77 & 9 & 30 & 2.11 & 12 & 30 & 4.39 & 9 & 34 & 28.88 \\
\hline & & & 6 & 14 & 38 & 0.39 & 14 & 37 & 0.46 & 13 & 38 & 23.04 & 10 & 31 & 3.33 & 14 & 31 & 3.82 & 13 & 38 & 32.54 \\
\hline & & 10 & 2 & 11 & 36 & 0.18 & 11 & 35 & 0.43 & 10 & 36 & 20.77 & 9 & 30 & 1.65 & 12 & 30 & 3.94 & 9 & 34 & 29.26 \\
\hline & & & 6 & 14 & 38 & 0.39 & 14 & 37 & 0.46 & 13 & 38 & 23.04 & 10 & 31 & 2.52 & 14 & 31 & 3.12 & 12 & 36 & 33.09 \\
\hline
\end{tabular}



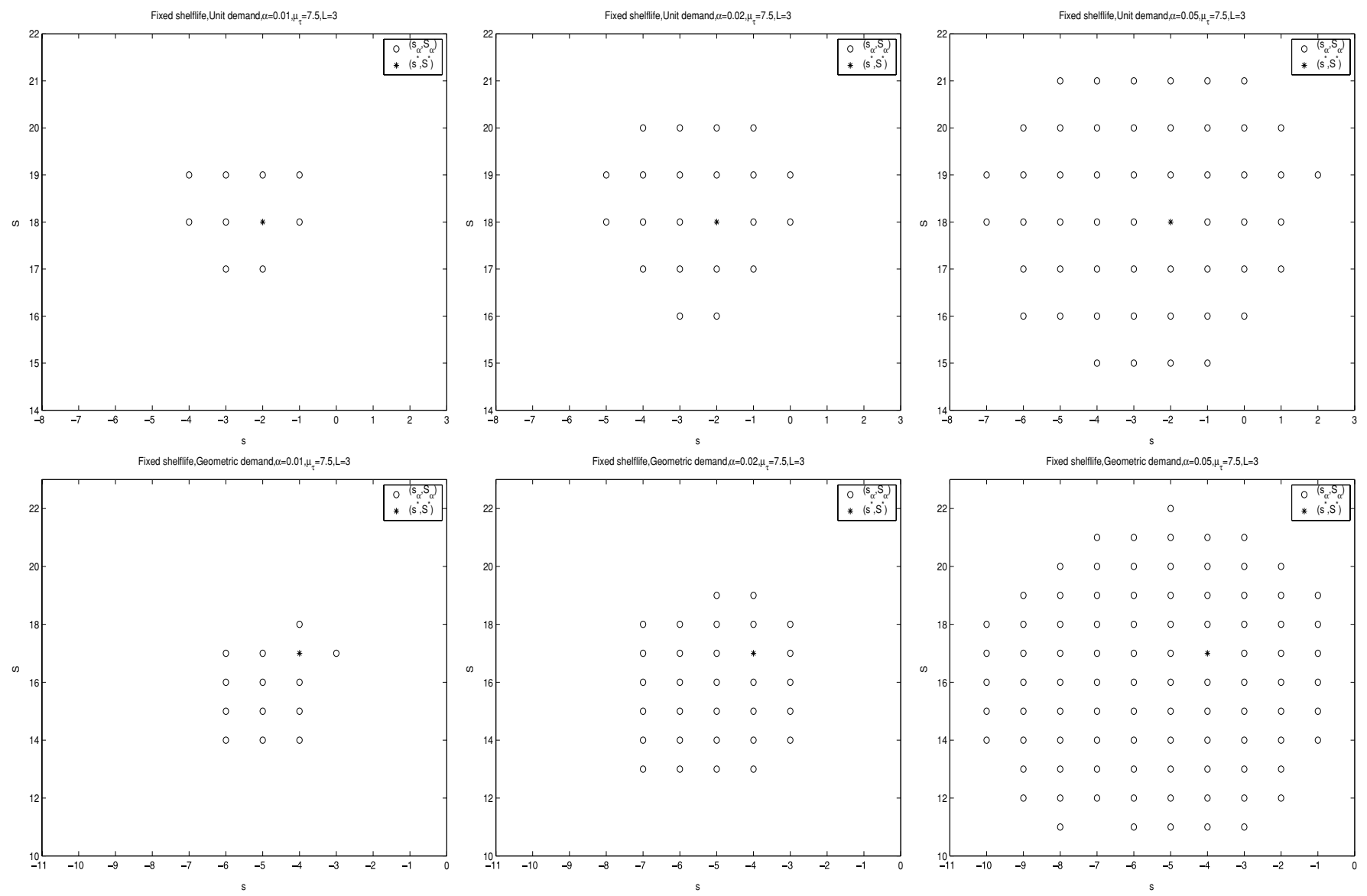

Fig. 5. Illustration of $\alpha \%$ bounds for positive lead time-fixed shelf life.

$\mathrm{H}_{2}$ which is also observed in the reported results of Lian and Liu (2001). For unit demands, out of 48 cases, $\mathrm{H}_{1}$ dominates $\mathrm{H}_{2}$ in 24 of them, $\mathrm{H}_{2}$ dominates $\mathrm{H}_{1}$ in 15 of them and in the remaining nine cases they perform equally well. Although it is hard to arrive at a general conclusion for this case, the proposed heuristic tends to behave better when the lead time is reasonably small compared to the mean shelf life. We also observe that both heuristics perform better for unit demands, where the average deviation from the optimal are 0.34 and $0.29 \%$ for $\mathrm{H}_{1}, \mathrm{H}_{2}$, respectively whereas the corresponding figures are 1.60 and $3.62 \%$ for geometric demand.

Another issue of interest is the sensitivity of the set of $(s, S)$ pairs to deviations from the optimal costs. In particular, we ask the following question: which set of $(s, S)$ values allows for at most an $\alpha \%$ deviation from the optimal cost? To this end, we obtained numerical results from the simulation model, from which we obtained the set of $\left(s_{\alpha}, S_{\alpha}\right)$ values that result in costs within $\alpha \%$ of the minimal cost. A large number of experiments for different choices of $\alpha, L$ values and shelf life and demand distributions are carried out and the results are displayed in Figs. 5 and 6 for $\alpha=1,2,5$ for fixed and gamma shelf lives respectively. We observe that the number of $\left(s_{\alpha}, S_{\alpha}\right)$ points increases significantly with $\alpha$ as expected. Furthermore, when the shelf life is random, the increase in this set is more notable, indicating a more flat function around $\left(s^{*}, S^{*}\right)$. Similarly, the cost rate function of the geometric demand is observed to be more flat than that of the unit demand. Finally, although it can not be generalized, an observation regarding the location of the optimal $\left(s^{*}, S^{*}\right)$ values is worth mentioning. For both fixed and random shelf lives, the $\left(s^{*}, S^{*}\right)$ pair tends to be placed toward the right side of the region constructed by $\left(s_{\alpha}, S_{\alpha}\right)$. This behavior may provide a better explanation for the performance of both heuristics. In particular, for positive lead times, both $\mathrm{H}_{1}$ and $\mathrm{H}_{2}$ add a non-negative quantity to the $s_{0}$ value obtained from the zero lead time model. The heuristic of Lian and Liu (2001) adds an overestimated quantity obtained as the expected perished units for the cycle, which probably pushed the modified $s_{0}$ value far beyond the optimal, whereas our heuristic adds a smaller quantity based on the remaining shelf life of the items, which possibly better estimates $s^{*}$ by inflating $s_{0}$ more appropriately and produces better results. 

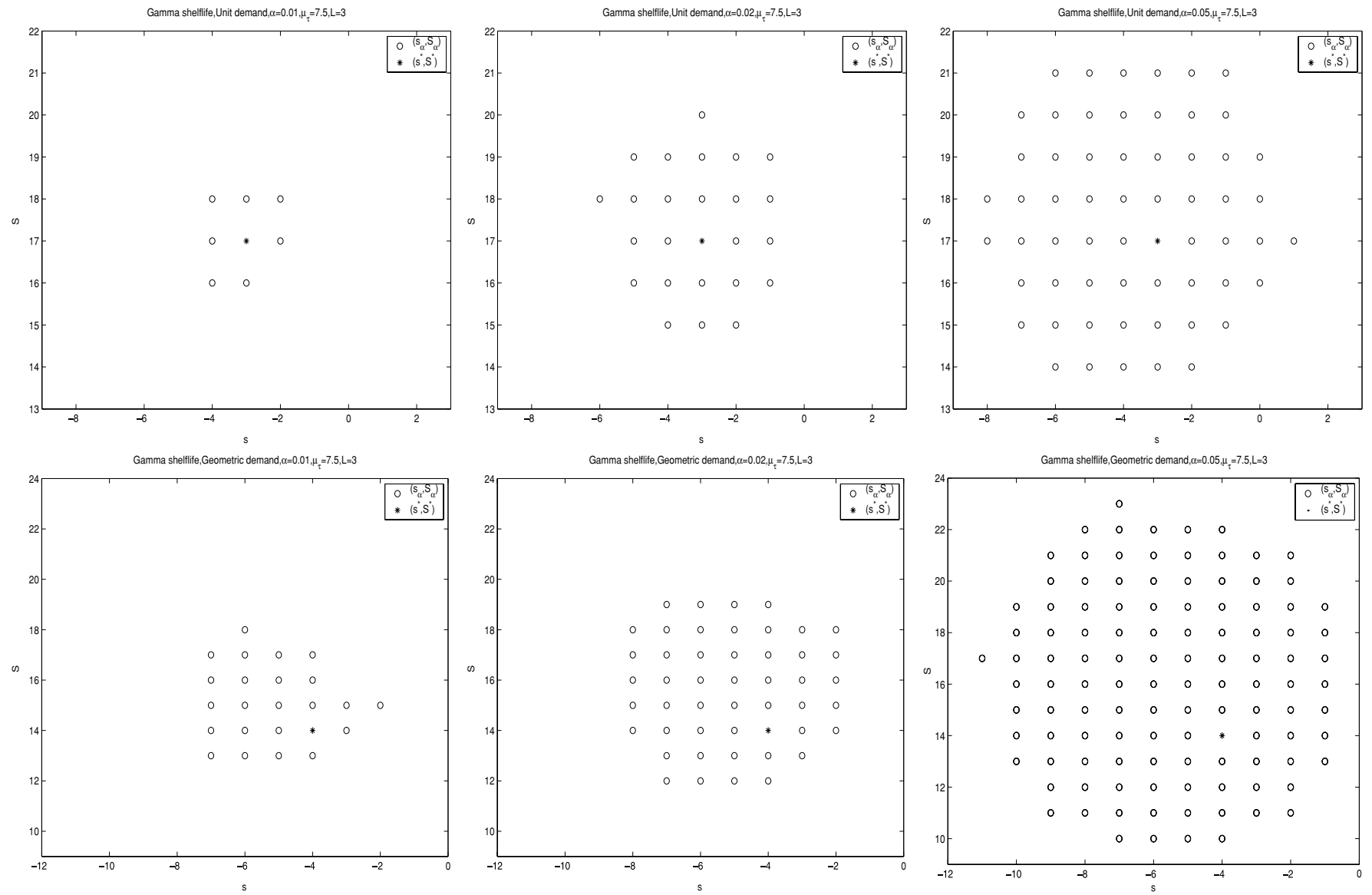

Fig. 6. Illustration of $\alpha \%$ bounds for positive lead time—gamma shelf life.

\section{Conclusions}

In this study, we considered a continuous review $(s, S)$-type policy for perishable goods with a random shelf life and renewal batch demands. Under the assumption of instantaneous replenishment, we developed a model for items with a general shelf life distribution, which is also suitable when the items in a batch can perish at a random time during their fixed shelf lives due to imperfect storage conditions. An explicit expression for the average cost rate function is obtained and the quasi-convexity of it is shown for unit demands. For positive lead times a heuristic is also proposed. An extensive numerical study is conducted to study the performance of the model and the suggested heuristic. We observed that significant reduction in the cost function is obtained by explicitly taking into account the randomness of the shelf life. Furthermore, we demonstrated that the system costs may differ drastically among various shelf life distributions, which implies that a precise estimation of the shelf life distribution is desirable. The numerical experiments indicated that the proposed heuristic for a positive lead time performs better than an available alternative in most of the tested cases with a pronounced difference for batch demands. Although both heuristics perform reason- ably well for unit demands, exact analysis for positive lead times still needs further research.

\section{References}

Avriel, M. (1976) Nonlinear Programming: Analysis and Methods, Prentice Hall, NJ.

Chiu, H.N. (1995) An approximation to the continuous review inventory model with perishable items and lead times. European Journal of Operational Research, 87, 93-108.

Cohen, M.A. (1976) Analysis of single critical number ordering policies for perishable inventories. Operations Research, 24, 726-741.

Fries, B. (1975) Optimal order policy for a perishable commodity with fixed lifetime. Operations Research, 23, 46-61.

Goyal, S.K. and Giri, B.C. (2001) Recent trends in modeling of deteriorating inventory. European Journal of Operational Research, 134,1-16.

Gürler, Ü. and Özkaya, B.Y. (2003) A note on "continuous review perishable inventory systems: models and heuristics". IIE Transactions, 35, 321-323.

Johnston, J.W., Hewett, E.W. and Hertog, M.L.A.T.M. (2002) Postharvest softening of apple (Malus domestica) fruit: a review. New Zealand Journal of Crop and Horticultural Science, 10, 145-160.

Kalpakam, S. and Arivarignan, G. (1988) A continuous review perishable inventory model. Statistics, 19, 389-398.

Kalpakam, S. and Sapna, K.P. (1994) Continuous review $\left(s_{y} S\right)$ inventory system with random lifetimes and positive leadtimes. Operations Research Letters, 16, 115-119. 
Kalpakam, S. and Sapna, K.P. (1996) A lost sales $(S-1, S)$ perishable inventory system with renewal demand. Naval Research Logistics, 43, 129-142.

Kalpakam, S. and Shanthi, S. (2001) A perishable inventory system with modified $(S-1, S)$ policy and arbitrary processing times. Computers \& Operations Research, 28, 453-471.

Lian, Z. and Liu, L. (2001) Continuous review perishable inventory systems: models and heuristics. IIE Transactions, 33, 809-822.

Liu, L. (1990) (s. S) continuous review inventory models for inventory with random lifetimes. Operations Research Letters, 9, 161-169.

Liu, L. and Lian, Z. (1999a) $(s, S)$ continuous review models for products with fixed lifetimes. Operations Research, 47, 150-158.

Liu, L. and Lian, Z. (1999) A discrete-time model for perishable inventory systems. Annals of Operations Research, 87, 103-116.

Liu, L. and Shi, D. (1999) An (s. S) model for inventory with exponential lifetimes and renewal demands. Naval Research Logistics, 46, 39-56.

Liu, L. and Yang, T. (1999) An (s. S) random lifetime inventory model with a positive lead time. European Journal of Operations Research, 113, 52-63.

Moorthy, A.K. Narasimhulu, Y.C. and Basha, I.R. (1992) On perishable inventory with markov chain demand quantities. International Journal of Information and Management Sciences, 3, 29-37.

Nahmias, S. (1975) Optimal ordering policies for perishable inventory-II. Operations Research, 23, 735-749.

Nahmias, S. (1976) Myopic approximations for the perishable inventory problem. Management Science, 22, 1002-1008.

Nahmias, S. (1977a) On ordering perishable inventory when both demand and lifetime are random. Management Science, 24, 82-90.

Nahmias, S. (1977b) Higher-order approximations for the perishable inventory problem. Operations Research, 25, 630-640.

Nahmias, S. (1982) Perishable inventory theory: a review. Operations Research, 30, 681-707.

Nahmias, S. and Pierskalla, W.P. (1975) Optimal ordering policies for a product that perishes in two periods subject to stochastic demand. Naval Research Logistics Quarterly, 20, 207-229.

Nahmias, S. and Wang, S.A. (1979) A heuristic lot size reorder point model for decaying inventories. Management Science, 25, 90-97.

Perry, D. and Posner, M.J.M. (1998) An $(S-1, S)$ inventory system with fixed shelf life and constant lead times. Operations Research, 46, S65-S71.

Raafat, F. (1991) Survey of literature on continuously deteriorating inventory models. Journal of the Operational Research Society, 42, 27-37.

Ravichandran, N. (1995) Stochastic analysis of a continuous review perishable inventory system with positive lead time and Poisson demands. European Journal of Operational Research, 84, $444-457$.

Ross, S. (1983) Stochastic Processes, Wiley, New York, NY.

Schmidt, C.P. and Nahmias, S. (1985) $(S-1, S)$ policies for perishable inventory. Management Science, 31, 719-728.

Tekin, E., Gürler, Ü. and Berk, E. (2001) Age based vs stock level control policies for a perishable inventory system. European Journal of Operations Research, 134, 81-101.

Van Zyl, G.J.J. (1964) Inventory control for perishable commodities. Ph.D. trans., University of North Carolina.

Weiss, H. (1980) Optimal ordering policies for continuous review perishable inventory models. Operations Research, 28, 365-374.

\section{Appendix}

We first present a result below needed for other derivations. The details of all proofs in this section can be obtained from the authors.

Lemma A1. Let $k$ be a non-negative integer. Then,

(i) $E\left[X_{k+1} I\left(X_{k}<\tau<X_{k+1}\right)\right]$

$$
=\int_{x=0}^{\infty} G(x)\left\{x \mathrm{~d} F_{k+1}(x)-(x+\mu) \mathrm{d} F_{k}(x)\right\},
$$

(ii) $E\left[X_{k} I\left(X_{k}<\tau<X_{k+1}\right)\right]$

$$
=\int_{x=0}^{\infty} x\left[W_{\tau-X_{1}}(x)-G(x)\right] \mathrm{d} F_{k}(x),
$$

(iii) $E\left[X_{k} I\left(X_{k+1}<\tau\right)\right]$

$$
\begin{aligned}
= & \int_{x=0}^{\infty} x \bar{W}_{\tau-X_{1}}(x) \mathrm{d} F_{k}(x), \\
\text { (iv) } & E\left[\tau I\left(X_{k}<\tau<X_{k+1}\right)\right] \\
= & \int_{x=0}^{\infty} x\left[F_{k}(x)-F_{k+1}(x)\right] \mathrm{d} G(x) .
\end{aligned}
$$

where $W_{\tau-X_{1}}(x)=\int_{t=0}^{\infty} G(x+t) \mathrm{d} F(t)$ is the d.f. of $\tau-X_{1}$, the remaining shelf life of a batch after the first demand.

Proof. Only the proofs of the first two parts will be provided, since the others follow similarly. We use the joint distribution function of $X_{k}, X_{k+1}$ and $\tau$ given as

$$
\begin{aligned}
& H_{X_{k}, X_{k+1}, \tau}(x, y, t) \\
& \quad=\int_{z=0}^{x} F(y-z) \mathrm{d} F_{k}(z) G(t) \quad x, y, t \geq 0, y>x .
\end{aligned}
$$

with

$$
\begin{aligned}
& \mathrm{d} H_{X_{k}, X_{k+1}, \tau}(x, y, t)=\mathrm{d} F_{k}(x) \mathrm{d} F(y-x) \mathrm{d} G(t) \\
& x, y, t \geq 0, y>x \text {. } \\
& \text { (i) } E\left[X_{k+1} I\left(X_{k}<\tau<X_{k+1}\right)\right]=\iiint_{x<t<y} y \mathrm{~d} H(x, y, t) \\
& =\iint_{x<y} y[G(y)-G(x)] \mathrm{d} F(y-x) \mathrm{d} F_{k}(x) \\
& =\int_{y=0}^{\infty} y G(y) \mathrm{d} F_{k+1}(y) \\
& -\int_{x=0}^{\infty} \int_{t=0}^{\infty}(x+t) G(x) \mathrm{d} F(t) \mathrm{d} F_{k}(x) \\
& =\int_{x=0}^{\infty} x G(x)\left[\mathrm{d} F_{k+1}(x)-\mathrm{d} F_{k}(x)\right]-\mu \int_{x=0}^{\infty} G(x) \mathrm{d} F_{k}(x) . \\
& \text { (ii) } E\left[X_{k} I\left(X_{k}<\tau<X_{k+1}\right)\right] \\
& =\iiint_{x<t<y} x \mathrm{~d} G(t) \mathrm{d} F(y-x) \mathrm{d} F_{k}(x) \\
& =\iint_{x<y} x[G(y)-G(x)] \mathrm{d} F(y-x) \mathrm{d} F_{k}(x) \\
& =\int_{x=0}^{\infty} x \int_{t=0}^{\infty} G(x+t) \mathrm{d} F(t) \mathrm{d} F_{k}(x)-\int_{x=0}^{\infty} x G(x) \mathrm{d} F_{k}(x) \\
& =\int_{x=0}^{\infty} x\left[W_{\tau-X_{1}}(x)-G(x)\right] \mathrm{d} F_{k}(x) .
\end{aligned}
$$

Proof of Lemma 1. The event $\left\{r_{1}=x\right\}$ is decomposed as disjoint events given by

$$
\begin{aligned}
& \left\{I_{X_{l_{0}}}=x, X_{l_{0}-1}<\tau, D_{l_{0}-1}=S\right\} \cup\left\{I_{X_{l_{0}}}=x, X_{l_{0}}\right. \\
& \left.\quad<\tau, D_{l_{0}-1}<S\right\} \cup\left\{I_{X_{N(\tau)+1}}=x, D_{N(\tau)}<S\right\} \\
& \quad \equiv A_{1} \cup A_{2} \cup A_{3},
\end{aligned}
$$


where $A_{1}$ corresponds to the realization where the inventory depletes by demand and hits exactly the zero level before perishing, $A_{2}$ corresponds to the event that the inventory drops below zero before perishing and without staying at the zero level and $A_{3}$ corresponds to the case where a positive number of items perish in a cycle.

$$
\begin{aligned}
P\left(A_{1}\right)= & \sum_{k} P\left(X_{k}<\tau, D_{k}=S, D_{k+1}=S-x\right) \\
= & v(-x)\left[\sum_{k=0}^{S} P\left(X_{k}<\tau\right) v_{k}(S)\right] \\
P\left(A_{2}\right)= & \sum_{k} P\left(X_{k}<\tau, D_{k-1} \leq S-1, D_{k}=S-x\right) \\
= & \sum_{k=1}^{S} P\left(X_{k}<\tau\right)\left[\sum_{i=k-1}^{S-1} v_{k-1}(i) v(S-i-x)\right] \\
P\left(A_{3}\right)= & \sum_{k} P\left(X_{k}<\tau<X_{k+1}, D_{k} \leq S-1, d_{k+1}=-x\right) \\
= & v(-x) \sum_{k=0}^{S-1} P\left(X_{k}<\tau<X_{k+1}\right) V_{k}(S-1), \\
P_{r_{1}}(x)= & P\left(A_{1}\right)+P\left(A_{2}\right)+P\left(A_{3}\right) \\
= & v(-x)\left[\sum_{k=0}^{S} P\left(X_{k}<\tau\right) v_{k}(S)\right] \\
& +\sum_{k=1}^{S} P\left(X_{k}<\tau\right)\left[\sum_{i=k-1}^{S-1} v_{k-1}(i) v(S-i-x)\right] \\
& +v(-x) \sum_{k=0}^{S-1} P\left(X_{k}<\tau<X_{k+1}\right) V_{k}(S-1) .
\end{aligned}
$$

Adjusting the indices of the summations and noting that $V_{S}(S-1)=0$ for $S \geq 0$, we obtain:

$$
\begin{aligned}
P_{r_{1}}(x)= & v(-x)\left[\sum_{k=0}^{S} P\left(X_{k}<\tau\right)\left[V_{k}(S)-V_{k}(S-1)\right]\right. \\
& \left.+\left[P\left(X_{k}<\tau\right)-P\left(X_{k+1}<\tau\right)\right] V_{k}(S-1)\right] \\
& +\sum_{k=1}^{S} P\left(X_{k}<\tau\right)\left[\sum_{i=k-1}^{S-1} v_{k-1}(i) v(S-i-x)\right] \\
= & v(-x)\left[\sum_{k=0}^{S} P\left(X_{k}<\tau\right) V_{k}(S)\right. \\
& \left.-\sum_{k=0}^{S} P\left(X_{k+1}<\tau\right) V_{k}(S-1)\right] \\
& +\sum_{k=1}^{S} P\left(X_{k}<\tau\right)\left[\sum_{i=k-1}^{S-1} v_{k-1}(i) v(S-i-x)\right] .
\end{aligned}
$$

The result follows by writing $P\left(X_{k}<\tau\right)=\int_{u=0}^{\infty} F_{k}(u) \mathrm{d} G(u)$.
Derivation of Equation (4). Referring to Equation (2), we have that:

$$
\begin{aligned}
E & {\left[X_{l_{0}} I\left(X_{l_{0}-1}<\tau, D_{l_{0}-1}=S\right)\right]=\sum_{k=0}^{S} E\left[X_{k+1} I\left(X_{k}<\tau\right)\right] v_{k}(S) } \\
= & \sum_{k=0}^{S} E\left[X_{k+1} I\left(X_{k}<\tau<X_{k+1}\right)\right]\left[V_{k}(S)-V_{k}(S-1)\right] \\
& \quad+\sum_{k=0}^{S} E\left[X_{k+1} I\left(X_{k+1}<\tau\right)\right], \\
E & {\left[X_{l_{0}} I\left(X_{l_{0}}<\tau, D_{l_{0}-1} \leq S-1\right)\right] } \\
= & \sum_{k=1}^{S} E\left[X_{k} I\left(X_{k}<\tau, D_{k-1} \leq S-1, D_{k}>S\right)\right] \\
= & \sum_{k=1}^{S} E\left[X_{k} I\left(X_{k}<\tau\right)\right]\left[V_{k-1}(S-1)-V_{k}(S)\right], \\
E & {\left[X_{N(\tau)+1} I\left(D_{N(\tau)} \leq S-1\right)\right] } \\
= & \sum_{k=0}^{S-1} E\left[X_{k+1} I\left(X_{k}<\tau<X_{k+1}, D_{k} \leq S-1\right)\right] \\
= & \sum_{k=0}^{S} E\left[X_{k+1} I\left(X_{k}<\tau\right)\right] V_{k}(S-1) \\
& -\sum_{k=0}^{S} E\left[X_{k} I\left(X_{k}<\tau\right)\right] V_{k}(S-1) .
\end{aligned}
$$

$E\left[C L_{1}(s, S)\right]$ is the sum of Equations (A1) to (A3). Application of Lemma A1 part (i) gives $E\left[C L_{1}(s, S)\right]$ as

$$
\begin{aligned}
E\left[C L_{1}(s, S)\right] & \sum_{k=0}^{S} V_{k}(S)\left\{\int_{x=0}^{\infty} x G(x)\left[\mathrm{d} F_{k+1}(x)-\mathrm{d} F_{k}(x)\right]\right. \\
& \left.-\mu \int_{x=0}^{\infty} G(x) \mathrm{d} F_{k}(x)\right\} \\
& +\sum_{k=1}^{S}\left\{v_{k}(S) \int_{x=0}^{\infty} x \bar{G}(x) \mathrm{d} F_{k+1}(x)+\left[V_{k-1}(S-1)\right.\right. \\
& \left.\left.-V_{k}(S)\right] \int_{x=0}^{\infty} x \bar{G}(x) \mathrm{d} F_{k}(x)\right\} .
\end{aligned}
$$

After some algebra, we write the above expression as

$$
\begin{aligned}
& \mu \sum_{k=1}^{S}\left[(k+1) v_{k}(S)+k V_{k-1}(S-1)-k V_{k}(S)\right] \\
& -\mu \sum_{k=0}^{S} V_{k}(S) \int_{x=0}^{\infty} G(x) \mathrm{d} F_{k}(x) \\
& +\mu v_{0}(S)+\sum_{k=0}^{S} V_{k}(S-1) \int_{x=0}^{\infty} x G(x) \mathrm{d} F_{k+1}(x) \\
& -\sum_{k=1}^{S} V_{k-1}(S-1) \int_{x=0}^{\infty} x G(x) \mathrm{d} F_{k}(x) .
\end{aligned}
$$


Noting that for $S>0, v_{0}(S)=0, V_{0}(S-1)=V_{0}(S)=1$ and

$$
\sum_{k=1}^{S}\left[(k+1) v_{k}(S)+k V_{k-1}(S-1)-k V_{k}(S)\right]=\sum_{k=0}^{S} V_{k}(S) \text {. }
$$

Also, for $S \geq 0$,

$$
\begin{aligned}
& \sum_{k=0}^{S} V_{k}(S-1) \int_{x=0}^{\infty} x G(x) \mathrm{d} F_{k+1}(x) \\
& -\sum_{k=1}^{S} V_{k-1}(S-1) \int_{x=0}^{\infty} x G(x) \mathrm{d} F_{k}(x)=0 .
\end{aligned}
$$

Hence, for $S>0$, we have that:

$$
\begin{aligned}
E & {\left[C L_{1}(s, S)\right] } \\
& =\mu \sum_{k=0}^{S} V_{k}(S)-\mu \sum_{k=0}^{S} V_{k}(S) \int_{x=0}^{\infty} G(x) \mathrm{d} F_{k}(x) \\
& =\mu \sum_{k=0}^{S} V_{k}(S) \int_{x=0}^{\infty} \bar{G}(x) \mathrm{d} F_{k}(x) .
\end{aligned}
$$

For $S=0, v_{0}(S)=1$ and the left-hand side of Equation (A4) is zero since the upper limit of the sum is greater than the lower one. In conjunction with Equation (A5) we conclude that for $S=0$ it also holds that:

$$
E\left[C L_{1}(s, S)\right]=\mu \sum_{k=0}^{S} V_{k}(S) \int_{x=0}^{\infty} \bar{G}(x) \mathrm{d} F_{k}(x) .
$$

Derivation of Equation (5). Let $p_{1}\left(k \mid r_{1}\right)$ be the probability that $n_{2}=k$ demands occur in subcycle 2 for a given $r_{1}$. Then

$$
p_{1}\left(k \mid r_{1}\right)=V_{k-1}\left(r_{1}-s-1\right)-V_{k}\left(r_{1}-s-1\right),
$$

and

$$
E\left[n_{2} \mid r_{1}\right]=\sum_{k=1}^{r_{1}-s} k p_{1}\left(k \mid r_{1}\right)=\sum_{k=0}^{r_{1}-s-1} V_{k}\left(r_{1}-s-1\right) .
$$

Then, $E\left[C L_{2}(s, S)\right]$ is given as follows:

$$
\begin{aligned}
& E\left[C L_{2}(s, S)\right] \\
& \quad=\sum_{x=s+1}^{-1} E\left[C L_{2}(s, S) \mid r_{1}=x\right] P_{r_{1}}(x) \\
& \quad=\mu \sum_{x=s+1}^{-1} P_{r_{1}}(x)\left[\sum_{k=0}^{x-s-1} V_{k}(x-s-1)\right] .
\end{aligned}
$$

\section{Derivation of Equation (9).}

$$
\begin{aligned}
E & {\left[H C_{i}\right] } \\
& =h \sum_{k=i-1}^{S-1}(S-k) v_{i-1}(k) E\left[\left(X_{i}-X_{i-1}\right) I\left(X_{i}<\tau\right)\right]
\end{aligned}
$$

$$
+h \sum_{k=i-1}^{S-1}(S-k) v_{i-1}(k) E\left[\left(\tau-X_{i-1}\right) I\left(X_{i-1}<\tau<X_{i}\right)\right] .
$$

Lemma $\mathrm{A} 1$ and the relation $\sum_{k=i-1}^{S-1}(S-k) v_{i-1}(k)=$ $\sum_{k=i-1}^{S-1} V_{i-1}(k)$ implies that:

$$
\begin{aligned}
E\left[H C_{i}\right]= & h \sum_{k=i-1}^{S-1} V_{i-1}(k)\left[\int_{u=0}^{\infty} u \bar{G}(u)\left[\mathrm{d} F_{i}(u)-\mathrm{d} F_{i-1}(u)\right]\right. \\
& \left.+\int_{u=0}^{\infty} u\left[\bar{F}_{i}(u)-\bar{F}_{i-1}(u)\right] \mathrm{d} G(u)\right]
\end{aligned}
$$

Finally, applying integration by parts to the first integral, we get:

$$
\begin{aligned}
& E\left[H C_{i}\right] \\
& =h \sum_{k=i-1}^{S-1} V_{i-1}(k)\left[\left.u \bar{G}(u)\left[F_{i}(u)-F_{i-1}(u)\right]\right|_{0} ^{\infty}\right. \\
& \left.\quad-\int_{u=0}^{\infty}\left[F_{i}(u)-F_{i-1}(u)\right][\bar{G}(u) \mathrm{d} u-u \mathrm{~d} G(u)]\right] \\
& \quad+h \sum_{k=i-1}^{S-1} V_{i-1}(k) \\
& \quad \times \int_{u=0}^{\infty} u\left[\bar{F}_{i}(u)-\bar{F}_{i-1}(u)\right] \mathrm{d} G(u) \\
& =h \sum_{k=i-1}^{S-1} V_{i-1}(k) \int_{u=0}^{\infty}\left[F_{i-1}(u)-F_{i}(u)\right] \bar{G}(u) \mathrm{d} u .
\end{aligned}
$$

\section{Derivation of Equations (10), (14) and (15).}

$$
\begin{aligned}
& E\left[P C_{i}\right] \\
& \quad=\pi E\left[\left(S-D_{i-1}\right) I\left(X_{i-1}<\tau \leq X_{i}, S-D_{i-1}>0\right)\right] \\
& \quad=\pi \sum_{k}^{S-1}(S-k) v_{i-1}(k) E\left[I\left(X_{i-1}<\tau \leq X_{i}\right)\right] \\
& \quad=\pi \sum_{k=i-1}^{S-1} V_{i-1}(k)\left[P\left(X_{i-1}<\tau\right)-P\left(X_{i}<\tau\right)\right] \\
& \quad=\pi \sum_{k=i-1}^{S-1} V_{i-1}(k) \int_{u=0}^{\infty}\left[F_{i-1}(u)-F_{i}(u)\right] \mathrm{d} G(u) . \\
& E\left[U S C_{i} \mid r_{1}\right] \\
& \quad=b E\left[\left(\tilde{D}_{i-1} I\left(\tilde{D}_{i-1}<r_{1}-s, \tilde{D}_{i} \geq r_{1}-s\right)\right]\right. \\
& \quad=b \sum_{k=i-1}^{r_{1}-s-1} k P\left(\tilde{d}_{i} \geq r_{1}-s-k\right) P\left(\tilde{D}_{i-1}=k\right) \\
& \quad=b \sum_{k=i-1}^{r_{1}-s-1} k \bar{V}\left(r_{1}-s-k-1\right) v_{i-1}(k) . \\
& E\left[T D S C_{i} \mid r_{1}\right] \\
& \quad=\rho E\left[( \tilde { X } _ { i } - \tilde { X } _ { i - 1 } ) ( \tilde { D } _ { i - 1 } - r _ { 1 } ) I \left(\tilde{D}_{i-1}\right.\right. \\
& \left.\left.\quad \leq r_{1}-s-1\right)\right]=\rho \mu \sum_{k=i-1}^{r_{1}-s-1}\left(k-r_{1}\right) v_{i-1}(k) .
\end{aligned}
$$


Results follow after summing over the index $i$.

Proof of Lemma 2. Let $\mathrm{d} x$ be sufficiently small such that $x+\mathrm{d} x<0$ :

$$
\begin{aligned}
\left\{r_{1} \in(x, x+\mathrm{d} x]\right\} & \equiv\left\{I_{X_{l_{0}}} \in(x, x+\mathrm{d} x], X_{l_{0}}<\tau, D_{l_{0}-1} \leq S\right\} \\
& \cup\left\{I_{X_{N(\tau)+1}} \in(x, x+\mathrm{d} x], D_{N(\tau)}<S\right\} \\
& \equiv A_{2} \cup A_{3} .
\end{aligned}
$$

Note that the event $A_{1}$ of discrete demand case drops here since its probability is zero:

$$
\begin{aligned}
P\left(A_{2}\right)= & \sum_{k=1}^{\infty} P\left(I_{X_{k}} \in(x, x+\mathrm{d} x], X_{k}<\tau, D_{k-1} \leq S\right) \\
= & \sum_{k=1}^{\infty}\left[\int_{u=0}^{S}[V(S-x-u+\mathrm{d} x)-V(S-x-u)]\right. \\
& \left.\times \mathrm{d} V_{k-1}(u)\right] \int_{z=0}^{\infty} \bar{G}(z) \mathrm{d} F_{k}(z) . \\
P\left(A_{3}\right)= & \sum_{k=1}^{\infty} P\left(X_{k}<\tau<X_{k+1}, D_{k}<S, I_{X_{k+1}} \in(x, x+\mathrm{d} x]\right) \\
= & {[V(-x)-V(-x-\mathrm{d} x)] \sum_{k=0}^{\infty} V_{k}(S) } \\
& \times \int_{z=0}^{\infty} \bar{G}(z)\left[\mathrm{d} F_{k}(z)-\mathrm{d} F_{k+1}(z)\right] .
\end{aligned}
$$

Applying integration by parts, we get:

$$
\begin{aligned}
P\left(r_{1}\right. & \in(x, x+\mathrm{d} x]) \\
= & \sum_{k=1}^{\infty} \int_{u=0}^{S}[V(S-x-u+\mathrm{d} x) \\
& -V(S-x-u)] \mathrm{d} V_{k-1}(u) \int_{z=0}^{\infty} F_{k}(z) \mathrm{d} G(z)+[V(-x) \\
& -V(-x-\mathrm{d} x)] \sum_{k=0}^{\infty} V_{k}(S) \int_{z=0}^{\infty}\left[F_{k}(z)-F_{k+1}(z)\right] \mathrm{d} G(z) .
\end{aligned}
$$

The result follows after dividing both sides by $\mathrm{d} x$ and taking the limit as $\mathrm{d} x \rightarrow 0$.

\section{Derivation of Equation (17).}

$$
\begin{aligned}
E & {\left[X_{l_{0}} I\left(X_{l_{0}}<\tau, D_{l_{0}-1} \leq S, D_{l_{0}}>S\right)\right] } \\
& =\sum_{k=1}^{\infty} E\left[X_{k} I\left(X_{k}<\tau, D_{k-1} \leq S, D_{k}>S\right)\right] \\
& =\sum_{k=1}^{\infty} E\left[X_{k} I\left(X_{k}<\tau\right)\right] P\left(D_{k-1} \leq S<D_{k}\right) \\
& =\sum_{k=1}^{\infty} E\left[X_{k} I\left(X_{k}<\tau\right)\right]\left[V_{k-1}(S)-V_{k}(S)\right] .
\end{aligned}
$$

$$
\begin{aligned}
E & {\left[X_{N(\tau)+1} I\left(X_{N(\tau)}<\tau<X_{N(\tau)+1}, D_{N(\tau)}<S\right)\right] } \\
& =\sum_{k=0}^{\infty} E\left[X_{k+1} I\left(X_{k}<\tau<X_{k+1}, D_{k}<S\right)\right] \\
& =\sum_{k=0}^{\infty} E\left[X_{k+1} I\left(X_{k}<\tau<X_{k+1}\right)\right] P\left(D_{k}<S\right) \\
& =\sum_{k=0}^{\infty} V_{k}(S)\left[E\left[X_{k+1} I\left(X_{k}<\tau\right)\right]-E\left[X_{k+1} I\left(X_{k+1}<\tau\right)\right]\right] .
\end{aligned}
$$

$E\left[C L_{1}(s, S)\right]$ is the sum of Equations (A7) and (A8) which is given by:

$$
\begin{aligned}
E\left[C L_{1}(s, S)\right]= & \sum_{k=1}^{\infty} E\left[X_{k+1}-X_{k} I\left(X_{k}<\tau\right)\right] V_{k}(S)+\mu V_{0}(S) \\
= & \sum_{k=1}^{\infty} \iiint_{x<t, x<y}(y-x) \mathrm{d} G(t) \mathrm{d} F(y-x) \\
& \times \mathrm{d} F_{k}(x)+\mu V_{0}(S) \\
= & \sum_{k=1}^{\infty} \int_{x=0}^{\infty} \int_{u=0}^{\infty} u \bar{G}(x) \mathrm{d} F(u) \mathrm{d} F_{k}(x)+\mu V_{0}(S) \\
= & \mu \sum_{k=1}^{\infty} \int_{x=0}^{\infty} \bar{G}(x) \mathrm{d} F_{k}(x)+\mu V_{0}(S) \\
= & \mu \sum_{k=0}^{\infty} \int_{x=0}^{\infty} \bar{G}(x) \mathrm{d} F_{k}(x),
\end{aligned}
$$

where the last equation follows from $\int_{x=0}^{\infty} \bar{G}(x) \mathrm{d} F_{0}(x)=1$. Integration by parts yields the result.

Derivation of Equation (18).

Noting that there is no upper limit for $n_{2}$ for continuous demand, we have that:

$$
\begin{aligned}
E\left[n_{2} \mid r_{1}\right] & =\sum_{k=1}^{\infty} k p_{1}\left(k \mid r_{1}\right)=\sum_{k=1}^{\infty} k\left[V_{k-1}\left(r_{1}-s\right)-V_{k}\left(r_{1}-s\right)\right] \\
& =\sum_{k=0}^{\infty} V_{k}\left(r_{1}-s\right) .
\end{aligned}
$$

Hence, $E\left[C L_{2}(s, S)\right]$ is given by:

$$
\begin{aligned}
E\left[C L_{2}(s, S)\right] & =\int_{x=s}^{0} E\left[C L_{2}(s, S) \mid r_{1}=x\right] \mathrm{d} F_{r_{1}}(x) \\
& =\mu \int_{x=s}^{0} E\left[n_{2} \mid x\right] \mathrm{d} F_{r_{1}}(x) \\
& =\mu \int_{x=s}^{0} \sum_{k=0}^{\infty} V_{k}(x-s) \mathrm{d} F_{r_{1}}(x) .
\end{aligned}
$$

Proof of Lemma 4. Suppose that $x^{*}$ is a strict local minimum of $\phi$ on $\mathcal{X}$. By Definition 1, there exists a subset 
$A=\left\{x:\left|x-x^{*}\right| \leq \epsilon, \epsilon>0\right\} \subset \mathcal{X}$ such that $\forall x \in A$ we have:

$$
\phi(x)>\phi\left(x^{*}\right) .
$$

Suppose that $x^{*}$ is not a strict global minimum. Then there exists an $\bar{x} \notin A$ such that:

$$
\phi(\bar{x}) \leq \phi\left(x^{*}\right) .
$$

For $\phi$ to be quasi-convex in $x, \forall \gamma \in[0,1]$ :

$$
\phi\left(\gamma \bar{x}+(1-\gamma) x^{*}\right) \leq \max \left(\phi(\bar{x}), \phi\left(x^{*}\right)\right) .
$$

Because there exists a $\gamma \in[0,1]$ such that $\gamma \bar{x}+(1-\gamma) x^{*} \in$ $A$, we have a contradiction for Equation (A9) by Equation (A11).

Proof of Lemma 5. We want to show $L_{1}(\gamma) \leq R_{1}(\gamma)$, where:

$$
\begin{aligned}
& L_{1}(\gamma)=\gamma E\left[C L\left(s_{1}, S_{1}\right)\right]+(1-\gamma) E\left[C L\left(s_{2}, S_{2}\right)\right], \\
& R_{1}(\gamma)=E\left[C L\left(\gamma s_{1}+(1-\gamma) s_{2}, \gamma S_{1}+(1-\gamma) S_{2}\right)\right] .
\end{aligned}
$$

where $\left(s_{1}, S_{1}\right),\left(s_{2}, S_{2}\right) \in\left(Z^{-} \times Z^{+}\right)$, and $\gamma \in[0,1]$ subject to $\left(\gamma s_{1}+(1-\gamma) s_{2}, \gamma S_{1}+(1-\gamma) S_{2}\right) \in\left(Z^{-}, Z^{+}\right)$. In order that $L_{1}(\gamma) \leq R_{1}(\gamma)$, we need to show that $L_{2}(\gamma) \leq R_{2}(\gamma)$ where:

$$
\begin{aligned}
L_{2}(\gamma)= & \gamma\left[\sum_{k=1}^{S_{1}} P\left(\tau>X_{k}\right)-\sum_{k=1}^{S_{2}} P\left(\tau>X_{k}\right)\right] \\
& +\sum_{k=1}^{S_{2}} P\left(\tau>X_{k}\right) \\
R_{2}(\gamma)= & \sum_{k=1}^{\gamma S_{1}+(1-\gamma) S_{2}} P\left(\tau>X_{k}\right) .
\end{aligned}
$$

Suppose $S_{1}>S_{2}$. Then,

$$
\begin{aligned}
& L_{2}(\gamma)=\gamma\left[\sum_{k=S_{2}+1}^{S_{1}} P\left(\tau>X_{k}\right)\right]+\sum_{k=1}^{S_{2}} P\left(\tau>X_{k}\right), \\
& R_{2}(\gamma)=\sum_{k=S_{2}+1}^{\gamma\left(S_{1}-S_{2}\right)+S_{2}} P\left(\tau>X_{k}\right)+\sum_{k=1}^{S_{2}} P\left(\tau>X_{k}\right) .
\end{aligned}
$$

When $\gamma=0,1 L_{2}(\gamma)=R_{2}(\gamma)$ holds and $L_{2}(\gamma)$ is increasing and linear in $\gamma$. To check the behavior of $R_{2}(\gamma)$, consider the difference $\Delta_{1}(\gamma)=R_{2}(\gamma+\epsilon)-R_{2}(\gamma)$ where $\epsilon=1 /\left(S_{1}-\right.$ $\left.S_{2}\right)>0$. Then,

$$
\begin{aligned}
\Delta_{1}(\gamma) & =\sum_{k=\gamma\left(S_{1}-S_{2}\right)+S_{2}+1}^{(\gamma+\epsilon)\left(S_{1}-S_{2}\right)+S_{2}} P\left(\tau>X_{k}\right), \\
\Delta_{1}(\gamma+\epsilon) & =\sum_{k=\gamma\left(S_{1}-S_{2}\right)+S_{2}+1}^{(\gamma+2 \epsilon)\left(S_{1}-S_{2}\right)+S_{2}} P\left(\tau>X_{k}\right) .
\end{aligned}
$$

Since $P\left(\tau>X_{k}\right)$ is increasing in $k$, we have $\Delta_{1}(\gamma+\epsilon)<$ $\Delta_{1}(\gamma)$ which means that $R_{2}(\gamma)$ is increasing and concave in $\gamma$. Combining the linearity of $L_{2}(\gamma)$ and concavity of $R_{2}(\gamma)$, we have $R_{2}(\gamma) \geq L_{2}(\gamma) \forall \gamma \in[0,1]$. The case $S_{1}<S_{2}$ is similar, except that $L_{2}(\gamma)$ is decreasing and linear and $R_{2}(\gamma)$ is decreasing and concave in $\gamma$. Then, $R_{2}(\gamma) \geq L_{2}(\gamma)$ holds $\forall \gamma \in[0,1]$. Finally $S_{1}=S_{2}$ implies $L_{2}(\gamma)=R_{2}(\gamma)$, which completes the proof of part (i). For part (ii), we see that $C_{1}(s)$ in Equation (19) is convex in $(s, S)$ since it is constant in $S$ and quadratic in $s$ with a non-negative leading coefficient. Since $\left\{X_{k}\right\}$ is a stochastically increasing sequence, $C_{2}(S)$ in Equation (20) is convex due to increasing firstorder difference. The convexity of $C_{3}(S)$ in Equation (21) follows from concavity of $\sum_{k=1}^{S} P\left(\tau>X_{k}\right)$.

\section{Biographies}

Ülkü Gürler is a Professor in the Industrial Engineering Department of Bilkent University, Ankara. She received her M.Sc. and Ph.D. degrees from the Wharton School of the University of Pennsylvania in Statistics. She has been a research scholar at the Institute of Statistics in Universite Catholique de Louvain and a visiting Professor at the Division of Statistic of University of California in Davis. Her research interests include stochastic modeling in inventory management, supply chain and reliability, Bayesian statistical analysis, and analysis of censored and truncated data.

Banu Yüksel Özkaya is a faculty member in the Department of Industrial Engineering at Hacettepe University, Ankara, Turkey. She received her B.S., M.S. and Ph.D. degrees in Industrial Engineering from Bilkent University, Ankara, Turkey. She worked as a postdoctoral fellow in the H. Milton Stewart School of Industrial and Systems Engineering at Georgia Institute of Technology. Her research focuses on inventory control problems, demand management through price and lead time decisions, competition between main and secondary markets in durable goods industry and behavioral effects of incentives in the automotive industry. 